\title{
Supporting Information \\ Mixed Substituted Porphyrins: Structural and \\ Electrochemical Redox Properties
}

P. Bhyrappa*, M. Sankar and B. Varghese

Department of Chemistry,

Indian Institute of Technology Madras,

Chennai - 600036.

S. No.

Table S1

Table S2

Table S3
Title

Bond Lengths $(\AA)$ of $\mathrm{CuTPP}(\mathrm{Ph})_{4}\left(\mathrm{CH}_{3}\right)_{4} \cdot 2 \mathrm{CHCl}_{3}$ Bond Angles ( $\left.{ }^{\circ}\right)$ of $\mathrm{Cu}\left(\mathrm{TPP}(\mathrm{Ph})\left(\mathrm{CH}_{3}\right)_{4} \cdot{ }^{\cdot} 2 \mathrm{CHCl}_{3}\right.$

Atomic Coordinates (x 104) and Equivalent Isotropic Displacement Parameters $\left(\AA^{2} \times 10^{3}\right)$ for $\mathrm{CuTPP}(\mathrm{Ph})_{4}\left(\mathrm{CH}_{3}\right)_{4} \cdot 2 \mathrm{CHCl}_{3}$
Page No

2

Table S7

Bond Lengths $(\AA)$ of NiTPP $(\mathrm{Ph})_{4}(\mathrm{CN})_{4}\left(\mathrm{C}_{5} \mathrm{H}_{5} \mathrm{~N}\right)_{2} \cdot 2\left(\mathrm{C}_{5} \mathrm{H}_{5} \mathrm{~N}\right)$.

Table S9 Atomic Coordinates (x 104) and Equivalent Isotropic Displacement Parameters $\left(\AA^{2 \mathrm{x} 103}\right)$ for $\operatorname{NiTPP}(\mathrm{Ph})_{4}(\mathrm{CN})_{4}\left(\mathrm{C}_{5} \mathrm{H}_{5} \mathrm{~N}\right)_{2} \cdot 2\left(\mathrm{C}_{5} \mathrm{H}_{5} \mathrm{~N}\right)$ 
Table S1 Bond Lengths $(\AA)$ of CuTPP $(\mathrm{Ph})_{4}\left(\mathrm{CH}_{3}\right)_{4} \cdot 2 \mathrm{CHCl}_{3}$.

\begin{tabular}{|c|c|c|c|}
\hline \multicolumn{2}{|c|}{ Bond Distance $(\AA)$} & \multirow{2}{*}{$\frac{\text { Bond }}{\mathrm{C}(21)-\mathrm{C}(22)}$} & \multirow{2}{*}{$\begin{array}{l}\text { Distance }(\AA) \\
1.388(5)\end{array}$} \\
\hline $\mathrm{C}(1)-\mathrm{N}(1)$ & $1.381(3)$ & & \\
\hline $\mathrm{C}(1)-\mathrm{C}(10) \# 1$ & $1.405(4)$ & $\mathrm{C}(21)-\mathrm{H}(21)$ & 0.9300 \\
\hline $\mathrm{C}(1)-\mathrm{C}(2)$ & $1.456(4)$ & $\mathrm{C}(22)-\mathrm{H}(22)$ & 0.9300 \\
\hline $\mathrm{C}(2)-\mathrm{C}(3)$ & $1.371(4)$ & $\mathrm{C}(23)-\mathrm{C}(28)$ & $1.380(4)$ \\
\hline$C(2)-C(11)$ & $1.478(4)$ & $\mathrm{C}(23)-\mathrm{C}(24)$ & $1.385(4)$ \\
\hline$C(3)-C(4)$ & $1.451(4)$ & $\mathrm{C}(24)-\mathrm{C}(25)$ & $1.384(4)$ \\
\hline C(3)-C(17) & $1.489(4)$ & $\mathrm{C}(24)-\mathrm{H}(24)$ & 0.9300 \\
\hline $\mathrm{C}(4)-\mathrm{N}(1)$ & $1.370(3)$ & $\mathrm{C}(25)-\mathrm{C}(26)$ & $1.376(5)$ \\
\hline$C(4)-C(5)$ & $1.414(4)$ & $\mathrm{C}(25)-\mathrm{H}(25)$ & 0.9300 \\
\hline$C(5)-C(6)$ & $1.402(4)$ & $\mathrm{C}(26)-\mathrm{C}(27)$ & $1.379(5)$ \\
\hline$C(5)-C(23)$ & $1.497(4)$ & $\mathrm{C}(26)-\mathrm{H}(26)$ & 0.9300 \\
\hline $\mathrm{C}(6)-\mathrm{N}(2)$ & $1.379(3)$ & $\mathrm{C}(27)-\mathrm{C}(28)$ & $1.392(4)$ \\
\hline$C(6)-C(7)$ & $1.452(4)$ & $\mathrm{C}(27)-\mathrm{H}(27)$ & 0.9300 \\
\hline $\mathrm{C}(7)-\mathrm{C}(8)$ & $1.363(4)$ & $\mathrm{C}(28)-\mathrm{H}(28)$ & 0.9300 \\
\hline $\mathrm{C}(7)-\mathrm{C}(35)$ & $1.505(4)$ & C(29)-C(30) & $1.385(4)$ \\
\hline $\mathrm{C}(8)-\mathrm{C}(9)$ & $1.452(4)$ & C(29)-C(34) & $1.389(4)$ \\
\hline $\mathrm{C}(8)-\mathrm{C}(36)$ & $1.501(4)$ & $\mathrm{C}(30)-\mathrm{C}(31)$ & $1.389(5)$ \\
\hline $\mathrm{C}(9)-\mathrm{N}(2)$ & $1.366(3)$ & $\mathrm{C}(30)-\mathrm{H}(30)$ & 0.9300 \\
\hline $\mathrm{C}(9)-\mathrm{C}(10)$ & $1.406(4)$ & C(31)-C(32) & $1.377(6)$ \\
\hline $\mathrm{C}(10)-\mathrm{C}(1) \# 1$ & $1.405(4)$ & $\mathrm{C}(31)-\mathrm{H}(31)$ & 0.9300 \\
\hline $\mathrm{C}(10)-\mathrm{C}(29)$ & $1.493(4)$ & $\mathrm{C}(32)-\mathrm{C}(33)$ & $1.377(6)$ \\
\hline $\mathrm{C}(11)-\mathrm{C}(16)$ & $1.387(4)$ & $\mathrm{C}(32)-\mathrm{H}(32)$ & 0.9300 \\
\hline $\mathrm{C}(11)-\mathrm{C}(12)$ & $1.392(4)$ & C(33)-C(34) & $1.371(5)$ \\
\hline $\mathrm{C}(12)-\mathrm{C}(13)$ & $1.383(5)$ & C(33)-H(33) & 0.9300 \\
\hline $\mathrm{C}(12)-\mathrm{H}(12)$ & 0.9300 & $\mathrm{C}(34)-\mathrm{H}(34)$ & 0.9300 \\
\hline $\mathrm{C}(13)-\mathrm{C}(14)$ & $1.378(7)$ & $\mathrm{C}(35)-\mathrm{H}(35 \mathrm{~A})$ & 0.9600 \\
\hline $\mathrm{C}(13)-\mathrm{H}(13)$ & 0.9300 & $\mathrm{C}(35)-\mathrm{H}(35 \mathrm{~B})$ & 0.9600 \\
\hline $\mathrm{C}(14)-\mathrm{C}(15)$ & $1.373(7)$ & $\mathrm{C}(35)-\mathrm{H}(35 \mathrm{C})$ & 0.9600 \\
\hline $\mathrm{C}(14)-\mathrm{H}(14)$ & 0.9300 & $\mathrm{C}(36)-\mathrm{H}(36 \mathrm{~A})$ & 0.9600 \\
\hline $\mathrm{C}(15)-\mathrm{C}(16)$ & $1.384(5)$ & $\mathrm{C}(36)-\mathrm{H}(36 \mathrm{~B})$ & 0.9600 \\
\hline $\mathrm{C}(15)-\mathrm{H}(15)$ & 0.9300 & $\mathrm{C}(36)-\mathrm{H}(36 \mathrm{C})$ & 0.9600 \\
\hline $\mathrm{C}(16)-\mathrm{H}(16)$ & 0.9300 & $\mathrm{C}(37)-\mathrm{Cl}(2)$ & $1.723(7)$ \\
\hline $\mathrm{C}(17)-\mathrm{C}(18)$ & $1.385(4)$ & $\mathrm{C}(37)-\mathrm{Cl}(3)$ & $1.732(6)$ \\
\hline $\mathrm{C}(17)-\mathrm{C}(22)$ & $1.388(4)$ & $\mathrm{C}(37)-\mathrm{Cl}(1)$ & $1.745(5)$ \\
\hline C(18)-C(19) & $1.383(5)$ & $\mathrm{C}(37)-\mathrm{H}(37)$ & 0.9800 \\
\hline $\mathrm{C}(18)-\mathrm{H}(18)$ & 0.9300 & $\mathrm{~N}(1)-\mathrm{Cu}$ & $1.962(2)$ \\
\hline C(19)-C(20) & $1.363(6)$ & $\mathrm{N}(2)-\mathrm{Cu}$ & $1.946(2)$ \\
\hline C(19)-H(19) & 0.9300 & $\mathrm{Cu}-\mathrm{N}(2) \# 1$ & $1.946(2)$ \\
\hline $\mathrm{C}(20)-\mathrm{C}(21)$ & $1.377(5)$ & Cu-N(1)\#1 & $1.962(2)$ \\
\hline $\mathrm{C}(20)-\mathrm{H}(20)$ & 0.9300 & & \\
\hline
\end{tabular}


Table S2 Bond Angles $\left(^{\circ}\right)$ of $\mathrm{CuTPP}(\mathrm{Ph})_{4}\left(\mathrm{CH}_{3}\right)_{4} \cdot 2 \mathrm{CHCl}_{3}$.

\begin{tabular}{|c|c|c|c|}
\hline Bond & Angle $\left(^{\circ}\right)$ & Bond & Angle $\left(^{\circ}\right)$ \\
\hline $\mathrm{N}(1)-\mathrm{C}(1)-\mathrm{C}(10) \# 1$ & $121.5(2)$ & $\mathrm{C}(14)-\mathrm{C}(13)-\mathrm{H}(13)$ & 120.1 \\
\hline $\mathrm{N}(1)-\mathrm{C}(1)-\mathrm{C}(2)$ & $108.7(2)$ & $\mathrm{C}(12)-\mathrm{C}(13)-\mathrm{H}(13)$ & 120.1 \\
\hline $\mathrm{C}(10) \# 1-\mathrm{C}(1)-\mathrm{C}(2)$ & $128.7(2)$ & $C(15)-C(14)-C(13)$ & $119.9(3)$ \\
\hline $\mathrm{C}(3)-\mathrm{C}(2)-\mathrm{C}(1)$ & $106.8(2)$ & $\mathrm{C}(15)-\mathrm{C}(14)-\mathrm{H}(14)$ & 120.0 \\
\hline $\mathrm{C}(3)-\mathrm{C}(2)-\mathrm{C}(11)$ & $125.5(2)$ & $\mathrm{C}(13)-\mathrm{C}(14)-\mathrm{H}(14)$ & 120.0 \\
\hline $\mathrm{C}(1)-\mathrm{C}(2)-\mathrm{C}(11)$ & $127.3(2)$ & $\mathrm{C}(14)-\mathrm{C}(15)-\mathrm{C}(16)$ & $120.3(4)$ \\
\hline$C(2)-C(3)-C(4)$ & $106.9(2)$ & $\mathrm{C}(14)-\mathrm{C}(15)-\mathrm{H}(15)$ & 119.8 \\
\hline $\mathrm{C}(2)-\mathrm{C}(3)-\mathrm{C}(17)$ & $124.7(2)$ & $\mathrm{C}(16)-\mathrm{C}(15)-\mathrm{H}(15)$ & 119.8 \\
\hline$C(4)-C(3)-C(17)$ & $127.9(2)$ & $\mathrm{C}(15)-\mathrm{C}(16)-\mathrm{C}(11)$ & $120.8(4)$ \\
\hline $\mathrm{N}(1)-\mathrm{C}(4)-\mathrm{C}(5)$ & $122.9(2)$ & $\mathrm{C}(15)-\mathrm{C}(16)-\mathrm{H}(16)$ & 119.6 \\
\hline $\mathrm{N}(1)-\mathrm{C}(4)-\mathrm{C}(3)$ & $109.4(2)$ & $\mathrm{C}(11)-\mathrm{C}(16)-\mathrm{H}(16)$ & 119.6 \\
\hline$C(5)-C(4)-C(3)$ & $127.5(2)$ & $\mathrm{C}(18)-\mathrm{C}(17)-\mathrm{C}(22)$ & $118.5(3)$ \\
\hline$C(6)-C(5)-C(4)$ & $122.8(2)$ & $\mathrm{C}(18)-\mathrm{C}(17)-\mathrm{C}(3)$ & 119.7(3) \\
\hline C(6)-C(5)-C(23) & $118.8(2)$ & $\mathrm{C}(22)-\mathrm{C}(17)-\mathrm{C}(3)$ & 121.8(3) \\
\hline $\mathrm{C}(4)-\mathrm{C}(5)-\mathrm{C}(23)$ & $118.4(2)$ & $\mathrm{C}(19)-\mathrm{C}(18)-\mathrm{C}(17)$ & $120.4(3)$ \\
\hline $\mathrm{N}(2)-\mathrm{C}(6)-\mathrm{C}(5)$ & $122.2(2)$ & C(19)-C(18)-H(18) & 119.8 \\
\hline $\mathrm{N}(2)-\mathrm{C}(6)-\mathrm{C}(7)$ & $108.8(2)$ & $\mathrm{C}(17)-\mathrm{C}(18)-\mathrm{H}(18)$ & 119.8 \\
\hline$C(5)-C(6)-C(7)$ & $127.9(2)$ & C(20)-C(19)-C(18) & $120.9(3)$ \\
\hline $\mathrm{C}(8)-\mathrm{C}(7)-\mathrm{C}(6)$ & $106.7(2)$ & C(20)-C(19)-H(19) & 119.5 \\
\hline $\mathrm{C}(8)-\mathrm{C}(7)-\mathrm{C}(35)$ & $124.5(3)$ & C(18)-C(19)-H(19) & 119.5 \\
\hline $\mathrm{C}(6)-\mathrm{C}(7)-\mathrm{C}(35)$ & 128.7(3) & C(19)-C(20)-C(21) & $119.3(3)$ \\
\hline $\mathrm{C}(7)-\mathrm{C}(8)-\mathrm{C}(9)$ & $107.4(2)$ & $\mathrm{C}(19)-\mathrm{C}(20)-\mathrm{H}(20)$ & 120.4 \\
\hline C(7)-C(8)-C(36) & 124.8(3) & $\mathrm{C}(21)-\mathrm{C}(20)-\mathrm{H}(20)$ & 120.4 \\
\hline $\mathrm{C}(9)-\mathrm{C}(8)-\mathrm{C}(36)$ & 127.5(3) & $\mathrm{C}(20)-\mathrm{C}(21)-\mathrm{C}(22)$ & $120.6(3)$ \\
\hline $\mathrm{N}(2)-\mathrm{C}(9)-\mathrm{C}(10)$ & $123.5(2)$ & $\mathrm{C}(20)-\mathrm{C}(21)-\mathrm{H}(21)$ & 119.7 \\
\hline $\mathrm{N}(2)-\mathrm{C}(9)-\mathrm{C}(8)$ & $109.0(2)$ & $\mathrm{C}(22)-\mathrm{C}(21)-\mathrm{H}(21)$ & 119.7 \\
\hline $\mathrm{C}(10)-\mathrm{C}(9)-\mathrm{C}(8)$ & $127.5(2)$ & $\mathrm{C}(21)-\mathrm{C}(22)-\mathrm{C}(17)$ & $120.2(3)$ \\
\hline $\mathrm{C}(1) \# 1-\mathrm{C}(10)-\mathrm{C}(9)$ & $122.1(2)$ & $\mathrm{C}(21)-\mathrm{C}(22)-\mathrm{H}(22)$ & 119.9 \\
\hline $\mathrm{C}(1) \# 1-\mathrm{C}(10)-\mathrm{C}(29)$ & $120.4(2)$ & $\mathrm{C}(17)-\mathrm{C}(22)-\mathrm{H}(22)$ & 119.9 \\
\hline$C(9)-C(10)-C(29)$ & $117.5(2)$ & $\mathrm{C}(28)-\mathrm{C}(23)-\mathrm{C}(24)$ & 119.3(3) \\
\hline$C(16)-C(11)-C(12)$ & 118.1(3) & $\mathrm{C}(28)-\mathrm{C}(23)-\mathrm{C}(5)$ & $119.6(2)$ \\
\hline$C(16)-C(11)-C(2)$ & $122.4(3)$ & $C(24)-C(23)-C(5)$ & 121.1(3) \\
\hline $\mathrm{C}(12)-\mathrm{C}(11)-\mathrm{C}(2)$ & 119.5(3) & $C(25)-C(24)-C(23)$ & $120.2(3)$ \\
\hline$C(13)-C(12)-C(11$ & 121.1(3) & $\mathrm{C}(25)-\mathrm{C}(24)-\mathrm{H}(24)$ & 119.9 \\
\hline $\mathrm{C}(13)-\mathrm{C}(12)-\mathrm{H}(12)$ & 119.4 & $\mathrm{C}(23)-\mathrm{C}(24)-\mathrm{H}(24)$ & 119.9 \\
\hline $\mathrm{C}(11)-\mathrm{C}(12)-\mathrm{H}(12)$ & 119.4 & $\mathrm{C}(26)-\mathrm{C}(25)-\mathrm{C}(24)$ & 120.3(3) \\
\hline $\mathrm{C}(14)-\mathrm{C}(13)-\mathrm{C}(12)$ & $119.7(4)$ & $\mathrm{C}(26)-\mathrm{C}(25)-\mathrm{H}(25)$ & 119.8 \\
\hline
\end{tabular}


Table S2

\begin{tabular}{|c|c|c|c|}
\hline Bond & Angle $\left(^{\circ}\right)$ & Bond & gle $\left({ }^{\circ}\right)$ \\
\hline $\mathrm{C}(24)-\mathrm{C}(25)-\mathrm{H}(25)$ & 119.8 & $\mathrm{C}(7)-\mathrm{C}(35)-\mathrm{H}(35 \mathrm{~B})$ & 109.5 \\
\hline $\mathrm{C}(25)-\mathrm{C}(26)-\mathrm{C}(27)$ & $120.0(3)$ & $\mathrm{H}(35 \mathrm{~A})-\mathrm{C}(35)-\mathrm{H}(35 \mathrm{~B})$ & 109.5 \\
\hline $\mathrm{C}(25)-\mathrm{C}(26)-\mathrm{H}(26)$ & 120.0 & $\mathrm{C}(7)-\mathrm{C}(35)-\mathrm{H}(35 \mathrm{C})$ & 109.5 \\
\hline $\mathrm{C}(27)-\mathrm{C}(26)-\mathrm{H}(26)$ & 120.0 & $\mathrm{H}(35 \mathrm{~A})-\mathrm{C}(35)-\mathrm{H}(35 \mathrm{C})$ & 109.5 \\
\hline $\mathrm{C}(26)-\mathrm{C}(27)-\mathrm{C}(28)$ & 119.7(3) & $\mathrm{H}(35 \mathrm{~B})-\mathrm{C}(35)-\mathrm{H}(35 \mathrm{C})$ & 109.5 \\
\hline $\mathrm{C}(26)-\mathrm{C}(27)-\mathrm{H}(27)$ & 120.1 & $\mathrm{C}(8)-\mathrm{C}(36)-\mathrm{H}(36 \mathrm{~A})$ & 109.5 \\
\hline $\mathrm{C}(28)-\mathrm{C}(27)-\mathrm{H}(27)$ & 120.1 & $\mathrm{C}(8)-\mathrm{C}(36)-\mathrm{H}(36 \mathrm{~B})$ & 109.5 \\
\hline $\mathrm{C}(23)-\mathrm{C}(28)-\mathrm{C}(27)$ & $120.4(3)$ & $\mathrm{H}(36 \mathrm{~A})-\mathrm{C}(36)-\mathrm{H}(36 \mathrm{~B})$ & 109.5 \\
\hline $\mathrm{C}(23)-\mathrm{C}(28)-\mathrm{H}(28)$ & 119.8 & $\mathrm{C}(8)-\mathrm{C}(36)-\mathrm{H}(36 \mathrm{C})$ & 109.5 \\
\hline $\mathrm{C}(27)-\mathrm{C}(28)-\mathrm{H}(28)$ & 119.8 & $\mathrm{H}(36 \mathrm{~A})-\mathrm{C}(36)-\mathrm{H}(36 \mathrm{C})$ & 109.5 \\
\hline $\mathrm{C}(30)-\mathrm{C}(29)-\mathrm{C}(34)$ & 118.8(3) & $\mathrm{H}(36 \mathrm{~B})-\mathrm{C}(36)-\mathrm{H}(36 \mathrm{C})$ & 109.5 \\
\hline $\mathrm{C}(30)-\mathrm{C}(29)-\mathrm{C}(10)$ & 118.9(3) & $\mathrm{Cl}(2)-\mathrm{C}(37)-\mathrm{Cl}(3)$ & 111.3(3) \\
\hline $\mathrm{C}(34)-\mathrm{C}(29)-\mathrm{C}(10)$ & 122.3(3) & $\mathrm{Cl}(2)-\mathrm{C}(37)-\mathrm{Cl}(1)$ & 109.5(3) \\
\hline $\mathrm{C}(29)-\mathrm{C}(30)-\mathrm{C}(31)$ & 120.1(3) & $\mathrm{Cl}(3)-\mathrm{C}(37)-\mathrm{Cl}(1)$ & $111.4(3)$ \\
\hline $\mathrm{C}(29)-\mathrm{C}(30)-\mathrm{H}(30)$ & 120.0 & $\mathrm{Cl}(2)-\mathrm{C}(37)-\mathrm{H}(37)$ & 108.2 \\
\hline $\mathrm{C}(31)-\mathrm{C}(30)-\mathrm{H}(30)$ & 120.0 & $\mathrm{Cl}(3)-\mathrm{C}(37)-\mathrm{H}(37)$ & 108.2 \\
\hline $\mathrm{C}(32)-\mathrm{C}(31)-\mathrm{C}(30)$ & $120.4(4)$ & $\mathrm{Cl}(1)-\mathrm{C}(37)-\mathrm{H}(37)$ & 108.2 \\
\hline $\mathrm{C}(32)-\mathrm{C}(31)-\mathrm{H}(31)$ & 119.8 & $\mathrm{C}(4)-\mathrm{N}(1)-\mathrm{C}(1)$ & $107.5(2)$ \\
\hline $\mathrm{C}(30)-\mathrm{C}(31)-\mathrm{H}(31)$ & 119.8 & $\mathrm{C}(4)-\mathrm{N}(1)-\mathrm{Cu}$ & $126.16(17)$ \\
\hline $\mathrm{C}(31)-\mathrm{C}(32)-\mathrm{C}(33)$ & 119.7(3) & $\mathrm{C}(1)-\mathrm{N}(1)-\mathrm{Cu}$ & $122.65(17)$ \\
\hline $\mathrm{C}(31)-\mathrm{C}(32)-\mathrm{H}(32)$ & 120.2 & $\mathrm{C}(9)-\mathrm{N}(2)-\mathrm{C}(6)$ & $107.6(2)$ \\
\hline $\mathrm{C}(33)-\mathrm{C}(32)-\mathrm{H}(32)$ & 120.2 & $\mathrm{C}(9)-\mathrm{N}(2)-\mathrm{Cu}$ & $125.75(18)$ \\
\hline $\mathrm{C}(34)-\mathrm{C}(33)-\mathrm{C}(32)$ & 120.2(3) & $\mathrm{C}(6)-\mathrm{N}(2)-\mathrm{Cu}$ & 124.51(17) \\
\hline $\mathrm{C}(34)-\mathrm{C}(33)-\mathrm{H}(33)$ & 119.9 & $\mathrm{~N}(2) \# 1-\mathrm{Cu}-\mathrm{N}(2)$ & $173.68(13)$ \\
\hline $\mathrm{C}(32)-\mathrm{C}(33)-\mathrm{H}(33)$ & 119.9 & $\mathrm{~N}(2) \# 1-\mathrm{Cu}-\mathrm{N}(1)$ & $90.04(9)$ \\
\hline $\mathrm{C}(33)-\mathrm{C}(34)-\mathrm{C}(29)$ & $120.9(3)$ & $\mathrm{N}(2)-\mathrm{Cu}-\mathrm{N}(1)$ & $90.27(9)$ \\
\hline $\mathrm{C}(33)-\mathrm{C}(34)-\mathrm{H}(34)$ & 119.5 & $\mathrm{~N}(2) \# 1-\mathrm{Cu}-\mathrm{N}(1) \# 1$ & $90.27(9)$ \\
\hline C(29)-C(34)-H(34) & 119.5 & $\mathrm{~N}(2)-\mathrm{Cu}-\mathrm{N}(1) \# 1$ & $90.04(9)$ \\
\hline $\mathrm{C}(7)-\mathrm{C}(35)-\mathrm{H}(35 \mathrm{~A})$ & 109.5 & $\mathrm{~N}(1)-\mathrm{Cu}-\mathrm{N}(1) \# 1$ & $174.37(12)$ \\
\hline
\end{tabular}


Table S3 Atomic Coordinates ( x $10^{4}$ ) and Equivalent Isotropic Displacement Parameters $\left(\AA^{2} \times 10^{3}\right)$ for CuTPP $(\mathrm{Ph})_{4}\left(\mathrm{CH}_{3}\right)_{4} \cdot 2 \mathrm{CHCl}_{3} \mathrm{U}(\mathrm{eq})$ is Defined as One Third of the Trace of the Orthogonalized Uij Tensor.

\begin{tabular}{|c|c|c|c|c|}
\hline & $\bar{x}$ & $\bar{y}$ & $\overline{\mathrm{z}}$ & $\mathrm{U}_{(\mathrm{eq})}$ \\
\hline $\mathrm{C}(1)$ & $11454(2)$ & $1004(1)$ & $3724(2)$ & $26(1)$ \\
\hline $\mathrm{C}(2)$ & $11641(2)$ & 766(1) & $4510(2)$ & $29(1)$ \\
\hline C(3) & $11073(2)$ & 1027(1) & $4825(2)$ & $28(1)$ \\
\hline $\mathrm{C}(4)$ & 10482(2) & 1355(1) & $4210(2)$ & $26(1)$ \\
\hline C(5) & $9743(2)$ & 1638(1) & $4240(2)$ & $27(1)$ \\
\hline C(6) & $9072(2)$ & 1685(1) & $3597(2)$ & $27(1)$ \\
\hline C(7) & $8259(2)$ & 1890(1) & $3572(2)$ & $33(1)$ \\
\hline $\mathrm{C}(8)$ & $7769(2)$ & 1668(1) & $2894(2)$ & $34(1)$ \\
\hline C(9) & $8279(2)$ & 1361(1) & $2477(2)$ & $28(1)$ \\
\hline $\mathrm{C}(10)$ & $8035(2)$ & 1053(1) & $1757(2)$ & $28(1)$ \\
\hline $\mathrm{C}(11)$ & $12250(2)$ & 269(1) & $4875(2)$ & $32(1)$ \\
\hline$C(12)$ & $12324(2)$ & $-298(2)$ & $4473(2)$ & $43(1)$ \\
\hline$C(13)$ & $12867(2)$ & $-785(2)$ & $4805(3)$ & $62(1)$ \\
\hline$C(14)$ & 13335(3) & $-714(2)$ & $5551(3)$ & $73(1)$ \\
\hline $\mathrm{C}(15)$ & $13269(2)$ & $-158(2)$ & $5956(2)$ & $66(1)$ \\
\hline$C(16)$ & $12730(2)$ & $330(2)$ & $5622(2)$ & $48(1)$ \\
\hline $\mathrm{C}(17)$ & 11032(2) & $900(1)$ & $5621(2)$ & $30(1)$ \\
\hline$C(18)$ & $10778(2)$ & $294(2)$ & $5808(2)$ & $42(1)$ \\
\hline C(19) & $10730(3)$ & $170(2)$ & $6543(2)$ & $57(1)$ \\
\hline$C(20)$ & 10954(3) & $632(2)$ & $7104(2)$ & $58(1)$ \\
\hline $\mathrm{C}(21)$ & $11233(2)$ & $1229(2)$ & $6934(2)$ & $52(1)$ \\
\hline$C(22)$ & $11274(2)$ & $1367(2)$ & $6196(2)$ & $40(1)$ \\
\hline C(23) & $9663(2)$ & 1865(1) & $5001(2)$ & $30(1)$ \\
\hline$C(24)$ & $9242(2)$ & $1498(2)$ & $5405(2)$ & $39(1)$ \\
\hline$C(25)$ & $9166(2)$ & $1720(2)$ & $6101(2)$ & $50(1)$ \\
\hline$C(26)$ & $9500(2)$ & $2310(2)$ & $6395(2)$ & $52(1)$ \\
\hline C(27) & $9916(2)$ & 2681(2) & $5996(2)$ & $50(1)$ \\
\hline$C(28)$ & $10004(2)$ & $2453(2)$ & $5301(2)$ & $39(1)$ \\
\hline C(29) & $7195(2)$ & 792(1) & $1504(2)$ & $33(1)$ \\
\hline C(30) & $6962(2)$ & $318(2)$ & $1945(2)$ & $46(1)$ \\
\hline C(31) & $6184(2)$ & $65(2)$ & $1721(3)$ & $62(1)$ \\
\hline C(32) & $5641(2)$ & $284(2)$ & $1060(3)$ & $64(1)$ \\
\hline C(33) & $5869(2)$ & $761(2)$ & $625(2)$ & $56(1)$ \\
\hline C(34) & $6635(2)$ & 1014(2) & $845(2)$ & $42(1)$ \\
\hline C(35) & $7984(2)$ & 2311(2) & $4133(2)$ & $51(1)$ \\
\hline C(36) & $6879(2)$ & 1798(2) & $2613(2)$ & $52(1)$ \\
\hline C(37) & $11225(3)$ & $2904(2)$ & $3334(3)$ & $87(2)$ \\
\hline
\end{tabular}


Table S3 (Contd.)

\begin{tabular}{|lrrll|}
\hline & $\mathrm{x}$ & $\mathrm{y}$ & \multicolumn{1}{c|}{$\mathrm{z}$} & \multicolumn{1}{c|}{$\mathrm{U}_{(\mathrm{eq})}$} \\
\hline $\mathrm{N}(1)$ & $10714(1)$ & $1314(1)$ & $3548(1)$ & $26(1)$ \\
$\mathrm{N}(2)$ & $9065(1)$ & $1414(1)$ & $2899(1)$ & $26(1)$ \\
$\mathrm{Cl}(1)$ & $11959(1)$ & $2720(1)$ & $4184(1)$ & $109(1)$ \\
$\mathrm{Cl}(2)$ & $10464(1)$ & $3352(2)$ & $3540(1)$ & $161(1)$ \\
$\mathrm{Cl}(3)$ & $11635(1)$ & $3317(1)$ & $2697(1)$ & $102(1)$ \\
$\mathrm{Cu}$ & 10000 & $1362(1)$ & 2500 & $23(1)$ \\
\hline
\end{tabular}


Table S4 Bond Lengths $(\AA)$ of $\mathrm{ZnTPP}(\mathrm{Ph})_{4} \mathrm{Br}_{4}\left(\mathrm{CH}_{3} \mathrm{OH}\right) \cdot \mathrm{CH}_{3} \mathrm{OH}$.

\begin{tabular}{|llll|}
\hline Bond & Distance $(\AA)$ & Bond & Distance $(\AA)$ \\
\hline $\mathrm{C}(1)-\mathrm{N}(1)$ & $1.373(10)$ & $\mathrm{C}(20)-\mathrm{C}(21)$ & $1.479(11)$ \\
$\mathrm{C}(1)-\mathrm{C}(20)$ & $1.421(11)$ & $\mathrm{C}(21)-\mathrm{C}(26)$ & $1.347(12)$ \\
$\mathrm{C}(1)-\mathrm{C}(2)$ & $1.434(12)$ & $\mathrm{C}(21)-\mathrm{C}(22)$ & $1.359(12)$ \\
$\mathrm{C}(2)-\mathrm{C}(3)$ & $1.392(11)$ & $\mathrm{C}(22)-\mathrm{C}(23)$ & $1.371(12)$ \\
$\mathrm{C}(2)-\mathrm{C}(27)$ & $1.505(12)$ & $\mathrm{C}(22)-\mathrm{H}(22)$ & 0.9300 \\
$\mathrm{C}(3)-\mathrm{C}(4)$ & $1.460(11)$ & $\mathrm{C}(23)-\mathrm{C}(24)$ & $1.371(13)$ \\
$\mathrm{C}(3)-\mathrm{C}(33)$ & $1.492(12)$ & $\mathrm{C}(23)-\mathrm{H}(23)$ & 0.9300 \\
$\mathrm{C}(4)-\mathrm{N}(1)$ & $1.413(10)$ & $\mathrm{C}(24)-\mathrm{C}(25)$ & $1.372(14)$ \\
$\mathrm{C}(4)-\mathrm{C}(5)$ & $1.449(11)$ & $\mathrm{C}(24)-\mathrm{H}(24)$ & 0.9300 \\
$\mathrm{C}(5)-\mathrm{C}(6)$ & $1.311(11)$ & $\mathrm{C}(25)-\mathrm{C}(26)$ & $1.416(13)$ \\
$\mathrm{C}(5)-\mathrm{C}(39)$ & $1.509(12)$ & $\mathrm{C}(25)-\mathrm{H}(25)$ & 0.9300 \\
$\mathrm{C}(6)-\mathrm{N}(2)$ & $1.392(10)$ & $\mathrm{C}(26)-\mathrm{H}(26)$ & 0.9300 \\
$\mathrm{C}(6)-\mathrm{C}(7)$ & $1.473(11)$ & $\mathrm{C}(27)-\mathrm{C}(28)$ & $1.345(12)$ \\
$\mathrm{C}(7)-\mathrm{C}(8)$ & $1.315(11)$ & $\mathrm{C}(27)-\mathrm{C}(32)$ & $1.361(12)$ \\
$\mathrm{C}(7)-\mathrm{Br}(1 \mathrm{~A})$ & $1.830(15)$ & $\mathrm{C}(28)-\mathrm{C}(29)$ & $1.364(13)$ \\
$\mathrm{C}(7)-\mathrm{Br}(1 \mathrm{~B})$ & $1.894(15)$ & $\mathrm{C}(28)-\mathrm{H}(28)$ & 0.9300 \\
$\mathrm{C}(8)-\mathrm{C}(9)$ & $1.496(12)$ & $\mathrm{C}(29)-\mathrm{C}(30)$ & $1.341(14)$ \\
$\mathrm{C}(8)-\mathrm{Br}(2 \mathrm{~A})$ & $1.867(15)$ & $\mathrm{C}(29)-\mathrm{H}(29)$ & 0.9300 \\
$\mathrm{C}(8)-\mathrm{Br}(2 \mathrm{~B})$ & $1.917(15)$ & $\mathrm{C}(30)-\mathrm{C}(31)$ & $1.395(14)$ \\
$\mathrm{C}(9)-\mathrm{C}(10)$ & $1.307(11)$ & $\mathrm{C}(30)-\mathrm{H}(30)$ & 0.9300 \\
$\mathrm{C}(9)-\mathrm{N}(2)$ & $1.388(10)$ & $\mathrm{C}(31)-\mathrm{C}(32)$ & $1.380(13)$ \\
$\mathrm{C}(10)-\mathrm{C}(11)$ & $1.448(11)$ & $\mathrm{C}(31)-\mathrm{H}(31)$ & 0.9300 \\
$\mathrm{C}(10)-\mathrm{C}(45)$ & $1.534(11)$ & $\mathrm{C}(32)-\mathrm{H}(32)$ & 0.9300 \\
$\mathrm{C}(11)-\mathrm{C}(12)$ & $1.410(12)$ & $\mathrm{C}(33)-\mathrm{C}(38)$ & $1.346(13)$ \\
$\mathrm{C}(11)-\mathrm{N}(3)$ & $1.454(10)$ & $\mathrm{C}(33)-\mathrm{C}(34)$ & $1.389(13)$ \\
$\mathrm{C}(12)-\mathrm{C}(13)$ & $1.358(11)$ & $\mathrm{C}(34)-\mathrm{C}(35)$ & $1.344(14)$ \\
$\mathrm{C}(12)-\mathrm{C}(51)$ & $1.487(12)$ & $\mathrm{C}(34)-\mathrm{H}(34)$ & 0.9300 \\
$\mathrm{C}(13)-\mathrm{C}(14)$ & $1.399(11)$ & $\mathrm{C}(35)-\mathrm{C}(36)$ & $1.353(15)$ \\
$\mathrm{C}(13)-\mathrm{C}(57)$ & $1.482(12)$ & $\mathrm{C}(35)-\mathrm{H}(35)$ & 0.9300 \\
$\mathrm{C}(14)-\mathrm{N}(3)$ & $1.365(10)$ & $\mathrm{C}(36)-\mathrm{C}(37)$ & $1.394(16)$ \\
$\mathrm{C}(14)-\mathrm{C}(15)$ & $1.479(12)$ & $\mathrm{C}(36)-\mathrm{H}(36)$ & 0.9300 \\
$\mathrm{C}(15)-\mathrm{C}(16)$ & $1.315(12)$ & $\mathrm{C}(37)-\mathrm{C}(38)$ & $1.387(14)$ \\
$\mathrm{C}(15)-\mathrm{C}(63)$ & $1.459(12)$ & $\mathrm{C}(37)-\mathrm{H}(37)$ & 0.9300 \\
$\mathrm{C}(16)-\mathrm{N}(4)$ & $1.431(11)$ & $\mathrm{C}(38)-\mathrm{H}(38)$ & 0.9300 \\
$\mathrm{C}(16)-\mathrm{C}(17)$ & $1.509(12)$ & $\mathrm{C}(39)-\mathrm{C}(40)$ & $1.405(13)$ \\
$\mathrm{C}(19)-\mathrm{C}(20)$ & $1.370(12)$ & $\mathrm{C}(39)-\mathrm{C}(44)$ & $1.405(13)$ \\
$\mathrm{C}(19)-\mathrm{N}(4)$ & $1.415(11)$ & $\mathrm{C}(40)-\mathrm{C}(41)$ & $1.342(14)$ \\
$\mathrm{C}(19)-\mathrm{C}(18)$ & $1.543(11)$ & $\mathrm{C}(40)-\mathrm{H}(40)$ & 0.9300 \\
& & & \\
\hline
\end{tabular}


Table S4 (Contd.).

\begin{tabular}{|c|c|c|c|}
\hline \multicolumn{2}{|c|}{ Bond Distance $(\AA)$} & \multirow{2}{*}{$\begin{array}{l}\text { Bond } \\
C(60)-C(61)\end{array}$} & \multirow{2}{*}{$\begin{array}{c}\text { Distance }(\AA) \\
1.354(13)\end{array}$} \\
\hline $\mathrm{C}(41)-\mathrm{C}(42)$ & $1.358(15)$ & & \\
\hline $\mathrm{C}(41)-\mathrm{H}(41)$ & 0.9300 & $\mathrm{C}(60)-\mathrm{H}(60)$ & 0.9300 \\
\hline$C(42)-C(43)$ & $1.381(15)$ & $\mathrm{C}(61)-\mathrm{C}(62)$ & $1.371(12)$ \\
\hline $\mathrm{C}(42)-\mathrm{H}(42)$ & 0.9300 & $\mathrm{C}(61)-\mathrm{H}(61)$ & 0.9300 \\
\hline$C(43)-C(44)$ & $1.380(13)$ & $\mathrm{C}(62)-\mathrm{H}(62)$ & 0.9300 \\
\hline $\mathrm{C}(43)-\mathrm{H}(43)$ & 0.9300 & $\mathrm{C}(63)-\mathrm{C}(64)$ & $1.339(12)$ \\
\hline $\mathrm{C}(44)-\mathrm{H}(44)$ & 0.9300 & $\mathrm{C}(63)-\mathrm{C}(68)$ & $1.408(12)$ \\
\hline$C(45)-C(50)$ & $1.333(12)$ & $\mathrm{C}(64)-\mathrm{C}(65)$ & $1.371(13)$ \\
\hline$C(45)-C(46)$ & $1.400(13)$ & $\mathrm{C}(64)-\mathrm{H}(64)$ & 0.9300 \\
\hline$C(46)-C(47)$ & 1.401(13) & $\mathrm{C}(65)-\mathrm{C}(66)$ & $1.395(13)$ \\
\hline $\mathrm{C}(46)-\mathrm{H}(46)$ & 0.9300 & $\mathrm{C}(65)-\mathrm{H}(65)$ & 0.9300 \\
\hline $\mathrm{C}(47)-\mathrm{C}(48)$ & $1.350(14)$ & $\mathrm{C}(66)-\mathrm{C}(67)$ & $1.379(13)$ \\
\hline $\mathrm{C}(47)-\mathrm{H}(47)$ & 0.9300 & $\mathrm{C}(66)-\mathrm{H}(66)$ & 0.9300 \\
\hline $\mathrm{C}(48)-\mathrm{C}(49)$ & $1.412(14)$ & $\mathrm{C}(67)-\mathrm{C}(68)$ & $1.362(12)$ \\
\hline $\mathrm{C}(48)-\mathrm{H}(48)$ & 0.9300 & $\mathrm{C}(67)-\mathrm{H}(67)$ & 0.9300 \\
\hline$C(49)-C(50)$ & $1.367(13)$ & $\mathrm{C}(68)-\mathrm{H}(68)$ & 0.9300 \\
\hline C(49)-H(49) & 0.9300 & $\mathrm{C}(69)-\mathrm{O}(1)$ & 1.38(3) \\
\hline $\mathrm{C}(50)-\mathrm{H}(50)$ & 0.9300 & $\mathrm{C}(69)-\mathrm{H}(69 \mathrm{~A})$ & 0.9600 \\
\hline $\mathrm{C}(51)-\mathrm{C}(52)$ & $1.359(12)$ & $\mathrm{C}(69)-\mathrm{H}(69 \mathrm{~B})$ & 0.9600 \\
\hline$C(51)-C(56)$ & $1.364(12)$ & $\mathrm{C}(69)-\mathrm{H}(69 \mathrm{C})$ & 0.9600 \\
\hline $\mathrm{C}(52)-\mathrm{C}(53)$ & $1.373(14)$ & $\mathrm{C}(70)-\mathrm{O}(1)$ & $1.29(3)$ \\
\hline $\mathrm{C}(52)-\mathrm{H}(52)$ & 0.9300 & $\mathrm{C}(70)-\mathrm{H}(70 \mathrm{~A})$ & 0.9600 \\
\hline $\mathrm{C}(53)-\mathrm{C}(54)$ & 1.391(15) & $\mathrm{C}(70)-\mathrm{H}(70 \mathrm{~B})$ & 0.9600 \\
\hline C(53)-H(53) & 0.9300 & $\mathrm{C}(70)-\mathrm{H}(70 \mathrm{C})$ & 0.9600 \\
\hline$C(54)-C(55)$ & $1.350(15)$ & $\mathrm{C}(71)-\mathrm{O}(2)$ & $1.57(2)$ \\
\hline $\mathrm{C}(54)-\mathrm{H}(54)$ & 0.9300 & $\mathrm{~N}(1)-\mathrm{Zn}$ & $1.976(7)$ \\
\hline$C(55)-C(56)$ & 1.361(13) & $\mathrm{N}(4)-\mathrm{Zn}$ & $2.072(7)$ \\
\hline $\mathrm{C}(55)-\mathrm{H}(55)$ & 0.9300 & $\mathrm{~N}(3)-\mathrm{Zn}$ & $2.025(7)$ \\
\hline $\mathrm{C}(56)-\mathrm{H}(56)$ & 0.9300 & $\mathrm{~N}(2)-\mathrm{Zn}$ & 2.131(7) \\
\hline $\mathrm{C}(57)-\mathrm{C}(62)$ & $1.377(12)$ & $\mathrm{O}(1)-\mathrm{Zn}$ & $2.044(8)$ \\
\hline C(57)-C(58) & $1.394(13)$ & $\operatorname{Br}(4 \mathrm{~A})-\mathrm{C}(18)$ & 1.891(13) \\
\hline C(58)-C(59) & $1.400(13)$ & $\mathrm{Br}(4 \mathrm{~B})-\mathrm{C}(18)$ & $1.868(13)$ \\
\hline C(58)-H(58) & 0.9300 & $\mathrm{Br}(3 \mathrm{~A})-\mathrm{C}(17)$ & $1.875(14)$ \\
\hline C(59)-C(60) & $1.378(14)$ & $\mathrm{Br}(3 \mathrm{~B})-\mathrm{C}(17)$ & $1.877(14)$ \\
\hline C(59)-H(59) & 0.9300 & $\mathrm{C}(18)-\mathrm{C}(17)$ & $1.273(11)$ \\
\hline
\end{tabular}


Table S5 Bond Angles $\left(^{\circ}\right)$ of $\mathrm{ZnTPP}(\mathrm{Ph})_{4} \mathrm{Br}_{4}\left(\mathrm{CH}_{3} \mathrm{OH}\right) \cdot \mathrm{CH}_{3} \mathrm{OH}$.

\begin{tabular}{|c|c|c|c|}
\hline Angle( & & Bond & Angle $\left(^{\circ}\right)$ \\
\hline$N(1)-C(1)-C(20)$ & 121.2(8) & $\mathrm{C}(10)-\mathrm{C}(11)-\mathrm{N}(3)$ & $120.9(7)$ \\
\hline $\mathrm{N}(1)-\mathrm{C}(1)-\mathrm{C}(2)$ & 113.7(8) & C(13)-C(12)-C(11) & 108.4(8) \\
\hline$C(20)-C(1)-C(2)$ & 124.8(9) & $\mathrm{C}(13)-\mathrm{C}(12)-\mathrm{C}(51)$ & 124.6(9) \\
\hline$C(3)-C(2)-C(1)$ & 106.8(8) & $\mathrm{C}(11)-\mathrm{C}(12)-\mathrm{C}(51)$ & 126.5(8) \\
\hline$C(3)-C(2)-C(27)$ & 122.7(8) & $\mathrm{C}(12)-\mathrm{C}(13)-\mathrm{C}(14)$ & 107.0(8) \\
\hline$C(1)-C(2)-C(27)$ & 128.8(8) & $\mathrm{C}(12)-\mathrm{C}(13)-\mathrm{C}(57)$ & $124.2(8)$ \\
\hline$C(2)-C(3)-C(4)$ & 104.4(8) & $\mathrm{C}(14)-\mathrm{C}(13)-\mathrm{C}(57)$ & 127.7(8) \\
\hline C(2)-C(3)-C(33) & 126.2(9) & N(3)-C(14)-C(13) & 112.5(8) \\
\hline$C(4)-C(3)-C(33)$ & 128.4(8) & $\mathrm{N}(3)-\mathrm{C}(14)-\mathrm{C}(15)$ & 118.9(8) \\
\hline $\mathrm{N}(1)-\mathrm{C}(4)-\mathrm{C}(5)$ & 122.2(7) & $\mathrm{C}(13)-\mathrm{C}(14)-\mathrm{C}(15)$ & 128.5(9) \\
\hline $\mathrm{N}(1)-\mathrm{C}(4)-\mathrm{C}(3)$ & 112.4(7) & $\mathrm{C}(16)-\mathrm{C}(15)-\mathrm{C}(63)$ & 119.7(9) \\
\hline$C(5)-C(4)-C(3)$ & 125.3(8) & C(16)-C(15)-C(14) & 125.2(9) \\
\hline$C(6)-C(5)-C(4)$ & 125.1(8) & C(63)-C(15)-C(14) & 115.1(8) \\
\hline$C(6)-C(5)-C(39)$ & 117.8(8) & $\mathrm{C}(15)-\mathrm{C}(16)-\mathrm{N}(4)$ & $124.4(9)$ \\
\hline$C(4)-C(5)-C(39)$ & 117.1(8) & $C(15)-C(16)-C(17$ & 132.4(9) \\
\hline $\mathrm{C}(5)-\mathrm{C}(6)-\mathrm{N}(2)$ & 121.8(8) & $\mathrm{N}(4)-\mathrm{C}(16)-\mathrm{C}(17)$ & 102.1(7) \\
\hline$C(5)-C(6)-C(7)$ & 133.0(9) & $\mathrm{C}(20)-\mathrm{C}(19)-\mathrm{N}(4)$ & 125.1(8) \\
\hline $\mathrm{N}(2)-\mathrm{C}(6)-\mathrm{C}(7)$ & 104.4(7) & C(20)-C(19)-C(18) & $133.0(8)$ \\
\hline $\mathrm{C}(8)-\mathrm{C}(7)-\mathrm{C}(6)$ & 109.0(8) & $\mathrm{N}(4)-\mathrm{C}(19)-\mathrm{C}(18)$ & 101.8(7) \\
\hline$C(8)-C(7)-B r(1 A)$ & 124.7(8) & $C(19)-C(20)-C(1)$ & 122.6(8) \\
\hline$C(6)-C(7)-B r(1 A)$ & 126.3(8) & $\mathrm{C}(19)-\mathrm{C}(20)-\mathrm{C}(21)$ & 119.3(8) \\
\hline $\mathrm{C}(8)-\mathrm{C}(7)-\mathrm{Br}(1 \mathrm{~B})$ & 124.7(8) & $\mathrm{C}(1)-\mathrm{C}(20)-\mathrm{C}(21)$ & 117.8(8) \\
\hline $\mathrm{C}(6)-\mathrm{C}(7)-\mathrm{Br}(1 \mathrm{~B})$ & 124.8(8) & $\mathrm{C}(26)-\mathrm{C}(21)-\mathrm{C}(22)$ & 116.1(9) \\
\hline $\operatorname{Br}(1 \mathrm{~A})-\mathrm{C}(7)-\mathrm{Br}(1 \mathrm{~B})$ & $11.3(6)$ & C(26)-C(21)-C(20) & 121.0(9) \\
\hline $\mathrm{C}(7)-\mathrm{C}(8)-\mathrm{C}(9)$ & 110.5(8) & C(22)-C(21)-C(20) & 122.8(9) \\
\hline$C(7)-C(8)-B r(2 A)$ & 126.1(8) & $\mathrm{C}(21)-\mathrm{C}(22)-\mathrm{C}(23)$ & $125.8(10)$ \\
\hline$C(9)-C(8)-B r(2 A)$ & 123.2(8) & $\mathrm{C}(21)-\mathrm{C}(22)-\mathrm{H}(22)$ & 117.1 \\
\hline$C(7)-C(8)-B r(2 B)$ & 122.5(8) & $\mathrm{C}(23)-\mathrm{C}(22)-\mathrm{H}(22)$ & 117.1 \\
\hline $\mathrm{C}(9)-\mathrm{C}(8)-\mathrm{Br}(2 \mathrm{~B})$ & 124.1(8) & $\mathrm{C}(24)-\mathrm{C}(23)-\mathrm{C}(22)$ & $116.5(10)$ \\
\hline $\operatorname{Br}(2 \mathrm{~A})-\mathrm{C}(8)-\mathrm{Br}(2 \mathrm{~B})$ & $12.3(5)$ & C(24)-C(23)-H(23) & 121.7 \\
\hline $\mathrm{C}(10)-\mathrm{C}(9)-\mathrm{N}(2)$ & $124.2(9)$ & $\mathrm{C}(22)-\mathrm{C}(23)-\mathrm{H}(23)$ & 121.7 \\
\hline $\mathrm{C}(10)-\mathrm{C}(9)-\mathrm{C}(8)$ & 132.4(9) & C(23)-C(24)-C(25) & $121.0(11)$ \\
\hline $\mathrm{N}(2)-\mathrm{C}(9)-\mathrm{C}(8)$ & 102.7(8) & $\mathrm{C}(23)-\mathrm{C}(24)-\mathrm{H}(24)$ & 119.5 \\
\hline C(9)-C(10)-C(11) & 125.5(8) & C(25)-C(24)-H(24) & 119.5 \\
\hline $\mathrm{C}(9)-\mathrm{C}(10)-\mathrm{C}(45)$ & 120.3(8) & C(24)-C(25)-C(26) & $118.6(11)$ \\
\hline $\mathrm{C}(11)-\mathrm{C}(10)-\mathrm{C}(45)$ & $114.0(8)$ & C(24)-C(25)-H(25) & 120.7 \\
\hline $\mathrm{C}(12)-\mathrm{C}(11)-\mathrm{C}(10)$ & 130.8(8) & $\mathrm{C}(26)-\mathrm{C}(25)-\mathrm{H}(25)$ & 120.7 \\
\hline $\mathrm{C}(12)-\mathrm{C}(11)-\mathrm{N}(3)$ & 108.2(8) & $\mathrm{C}(21)-\mathrm{C}(26)-\mathrm{C}(25)$ & $121.7(10)$ \\
\hline
\end{tabular}


Table S5 (Contd.).

\begin{tabular}{|c|c|c|c|}
\hline \multicolumn{2}{|c|}{ Angle $\left(^{\circ}\right)$} & Bond & Angle $\left(^{\circ}\right)$ \\
\hline $\mathrm{C}(21)-\mathrm{C}(26)-\mathrm{H}(26)$ & 119.2 & $\mathrm{C}(40)-\mathrm{C}(39)-\mathrm{C}(44)$ & $117.5(10)$ \\
\hline $\mathrm{C}(25)-\mathrm{C}(26)-\mathrm{H}(26)$ & 119.2 & $\mathrm{C}(40)-\mathrm{C}(39)-\mathrm{C}(5)$ & $121.6(9)$ \\
\hline$C(28)-C(27)-C(32)$ & 119.9(10) & $\mathrm{C}(44)-\mathrm{C}(39)-\mathrm{C}(5)$ & 120.9(9) \\
\hline$C(28)-C(27)-C(2)$ & 117.9(9) & $\mathrm{C}(41)-\mathrm{C}(40)-\mathrm{C}(39)$ & $120.4(12)$ \\
\hline $\mathrm{C}(32)-\mathrm{C}(27)-\mathrm{C}(2)$ & 121.9(9) & $\mathrm{C}(41)-\mathrm{C}(40)-\mathrm{H}(40)$ & 119.8 \\
\hline $\mathrm{C}(27)-\mathrm{C}(28)-\mathrm{C}(29)$ & $121.6(11)$ & $\mathrm{C}(39)-\mathrm{C}(40)-\mathrm{H}(40)$ & 119.8 \\
\hline $\mathrm{C}(27)-\mathrm{C}(28)-\mathrm{H}(28)$ & 119.2 & $\mathrm{C}(40)-\mathrm{C}(41)-\mathrm{C}(42)$ & $121.5(13)$ \\
\hline $\mathrm{C}(29)-\mathrm{C}(28)-\mathrm{H}(28)$ & 119.2 & $\mathrm{C}(40)-\mathrm{C}(41)-\mathrm{H}(41)$ & 119.3 \\
\hline$C(30)-C(29)-C(28)$ & 119.1(12) & $\mathrm{C}(42)-\mathrm{C}(41)-\mathrm{H}(41)$ & 119.3 \\
\hline C(30)-C(29)-H(29) & 120.4 & $\mathrm{C}(41)-\mathrm{C}(42)-\mathrm{C}(43)$ & $121.2(13)$ \\
\hline C(28)-C(29)-H(29) & 120.4 & $\mathrm{C}(41)-\mathrm{C}(42)-\mathrm{H}(42)$ & 119.4 \\
\hline$C(29)-C(30)-C(31)$ & $121.0(12)$ & $\mathrm{C}(43)-\mathrm{C}(42)-\mathrm{H}(42)$ & 119.4 \\
\hline C(29)-C(30)-H(30) & 119.5 & $\mathrm{C}(44)-\mathrm{C}(43)-\mathrm{C}(42)$ & $117.9(12)$ \\
\hline $\mathrm{C}(31)-\mathrm{C}(30)-\mathrm{H}(30)$ & 119.5 & $\mathrm{C}(44)-\mathrm{C}(43)-\mathrm{H}(43)$ & 121.0 \\
\hline C(32)-C(31)-C(30) & 118.1(11) & $\mathrm{C}(42)-\mathrm{C}(43)-\mathrm{H}(43)$ & 121.0 \\
\hline $\mathrm{C}(32)-\mathrm{C}(31)-\mathrm{H}(31)$ & 120.9 & $\mathrm{C}(43)-\mathrm{C}(44)-\mathrm{C}(39)$ & $121.5(11)$ \\
\hline $\mathrm{C}(30)-\mathrm{C}(31)-\mathrm{H}(31)$ & 120.9 & $\mathrm{C}(43)-\mathrm{C}(44)-\mathrm{H}(44)$ & 119.2 \\
\hline C(27)-C(32)-C(31) & $120.1(11)$ & C(39)-C(44)-H(44) & 119.2 \\
\hline $\mathrm{C}(27)-\mathrm{C}(32)-\mathrm{H}(32)$ & 119.9 & $C(50)-C(45)-C(46)$ & $120.7(10)$ \\
\hline $\mathrm{C}(31)-\mathrm{C}(32)-\mathrm{H}(32)$ & 119.9 & $\mathrm{C}(50)-\mathrm{C}(45)-\mathrm{C}(10)$ & $124.5(9)$ \\
\hline C(38)-C(33)-C(34) & $116.4(10)$ & $\mathrm{C}(46)-\mathrm{C}(45)-\mathrm{C}(10)$ & 114.8(9) \\
\hline C(38)-C(33)-C(3) & $120.7(10)$ & $\mathrm{C}(45)-\mathrm{C}(46)-\mathrm{C}(47)$ & $116.0(10)$ \\
\hline C(34)-C(33)-C(3) & $122.9(10)$ & $\mathrm{C}(45)-\mathrm{C}(46)-\mathrm{H}(46)$ & 122.0 \\
\hline C(35)-C(34)-C(33) & 119.1(11) & $\mathrm{C}(47)-\mathrm{C}(46)-\mathrm{H}(46)$ & 122.0 \\
\hline $\mathrm{C}(35)-\mathrm{C}(34)-\mathrm{H}(34)$ & 120.4 & $\mathrm{C}(48)-\mathrm{C}(47)-\mathrm{C}(46)$ & $123.0(11)$ \\
\hline C(33)-C(34)-H(34) & 120.4 & $\mathrm{C}(48)-\mathrm{C}(47)-\mathrm{H}(47)$ & 118.5 \\
\hline C(34)-C(35)-C(36) & $124.3(14)$ & $\mathrm{C}(46)-\mathrm{C}(47)-\mathrm{H}(47)$ & 118.5 \\
\hline $\mathrm{C}(34)-\mathrm{C}(35)-\mathrm{H}(35)$ & 117.9 & $\mathrm{C}(47)-\mathrm{C}(48)-\mathrm{C}(49)$ & $119.5(11)$ \\
\hline $\mathrm{C}(36)-\mathrm{C}(35)-\mathrm{H}(35)$ & 117.9 & $\mathrm{C}(47)-\mathrm{C}(48)-\mathrm{H}(48)$ & 120.3 \\
\hline C(35)-C(36)-C(37) & $118.5(14)$ & $\mathrm{C}(49)-\mathrm{C}(48)-\mathrm{H}(48)$ & 120.3 \\
\hline $\mathrm{C}(35)-\mathrm{C}(36)-\mathrm{H}(36)$ & 120.7 & $\mathrm{C}(50)-\mathrm{C}(49)-\mathrm{C}(48)$ & $117.0(11)$ \\
\hline $\mathrm{C}(37)-\mathrm{C}(36)-\mathrm{H}(36)$ & 120.7 & $\mathrm{C}(50)-\mathrm{C}(49)-\mathrm{H}(49)$ & 121.5 \\
\hline C(38)-C(37)-C(36) & 115.7(13) & $\mathrm{C}(48)-\mathrm{C}(49)-\mathrm{H}(49)$ & 121.5 \\
\hline C(38)-C(37)-H(37) & 122.1 & $\mathrm{C}(45)-\mathrm{C}(50)-\mathrm{C}(49)$ & 123.8(11) \\
\hline $\mathrm{C}(36)-\mathrm{C}(37)-\mathrm{H}(37)$ & 122.1 & $\mathrm{C}(45)-\mathrm{C}(50)-\mathrm{H}(50)$ & 118.1 \\
\hline C(33)-C(38)-C(37) & $125.8(12)$ & $\mathrm{C}(49)-\mathrm{C}(50)-\mathrm{H}(50)$ & 118.1 \\
\hline C(33)-C(38)-H(38) & 117.1 & $\mathrm{C}(52)-\mathrm{C}(51)-\mathrm{C}(56)$ & $115.7(10)$ \\
\hline C(37)-C(38)-H(38) & 117.1 & $\mathrm{C}(52)-\mathrm{C}(51)-\mathrm{C}(12)$ & 120.9(9) \\
\hline
\end{tabular}


Table S5 (Contd.).

\begin{tabular}{|c|c|c|c|}
\hline Bond & Angle $\left(^{\circ}\right)$ & Bond & Angle $\left(^{\circ}\right)$ \\
\hline C(56)-C(51)-C(12) & 123.2(9) & $\mathrm{C}(63)-\mathrm{C}(64)-\mathrm{H}(64)$ & 116.4 \\
\hline $\mathrm{C}(51)-\mathrm{C}(52)-\mathrm{C}(53)$ & 124.1(11) & $\mathrm{C}(65)-\mathrm{C}(64)-\mathrm{H}(64)$ & 116.4 \\
\hline $\mathrm{C}(51)-\mathrm{C}(52)-\mathrm{H}(52)$ & 118.0 & $\mathrm{C}(64)-\mathrm{C}(65)-\mathrm{C}(66)$ & $114.7(11)$ \\
\hline $\mathrm{C}(53)-\mathrm{C}(52)-\mathrm{H}(52)$ & 118.0 & $\mathrm{C}(64)-\mathrm{C}(65)-\mathrm{H}(65)$ & 122.7 \\
\hline C(52)-C(53)-C(54) & 117.7(12) & $\mathrm{C}(66)-\mathrm{C}(65)-\mathrm{H}(65)$ & 122.7 \\
\hline $\mathrm{C}(52)-\mathrm{C}(53)-\mathrm{H}(53)$ & 121.2 & $\mathrm{C}(67)-\mathrm{C}(66)-\mathrm{C}(65)$ & $123.0(11)$ \\
\hline $\mathrm{C}(54)-\mathrm{C}(53)-\mathrm{H}(53)$ & 121.2 & $\mathrm{C}(67)-\mathrm{C}(66)-\mathrm{H}(66)$ & 118.5 \\
\hline $\mathrm{C}(55)-\mathrm{C}(54)-\mathrm{C}(53)$ & 119.2(12) & $\mathrm{C}(65)-\mathrm{C}(66)-\mathrm{H}(66)$ & 118.5 \\
\hline $\mathrm{C}(55)-\mathrm{C}(54)-\mathrm{H}(54)$ & 120.4 & $\mathrm{C}(68)-\mathrm{C}(67)-\mathrm{C}(66)$ & 116.7(11) \\
\hline C(53)-C(54)-H(54) & 120.4 & $\mathrm{C}(68)-\mathrm{C}(67)-\mathrm{H}(67)$ & 121.7 \\
\hline $\mathrm{C}(54)-\mathrm{C}(55)-\mathrm{C}(56)$ & $120.6(12)$ & $\mathrm{C}(66)-\mathrm{C}(67)-\mathrm{H}(67)$ & 121.7 \\
\hline $\mathrm{C}(54)-\mathrm{C}(55)-\mathrm{H}(55)$ & 119.7 & $\mathrm{C}(67)-\mathrm{C}(68)-\mathrm{C}(63)$ & $123.9(10)$ \\
\hline $\mathrm{C}(56)-\mathrm{C}(55)-\mathrm{H}(55)$ & 119.7 & $\mathrm{C}(67)-\mathrm{C}(68)-\mathrm{H}(68)$ & 118.0 \\
\hline $\mathrm{C}(55)-\mathrm{C}(56)-\mathrm{C}(51)$ & 122.7(11) & $\mathrm{C}(63)-\mathrm{C}(68)-\mathrm{H}(68)$ & 118.0 \\
\hline $\mathrm{C}(55)-\mathrm{C}(56)-\mathrm{H}(56)$ & 118.7 & $\mathrm{O}(1)-\mathrm{C}(69)-\mathrm{H}(69 \mathrm{~A})$ & 109.5 \\
\hline $\mathrm{C}(51)-\mathrm{C}(56)-\mathrm{H}(56)$ & 118.7 & $\mathrm{O}(1)-\mathrm{C}(69)-\mathrm{H}(69 \mathrm{~B})$ & 109.5 \\
\hline $\mathrm{C}(62)-\mathrm{C}(57)-\mathrm{C}(58)$ & 117.6(9) & $\mathrm{H}(69 \mathrm{~A})-\mathrm{C}(69)-\mathrm{H}(69 \mathrm{~B})$ & 109.5 \\
\hline C(62)-C(57)-C(13) & $122.2(9)$ & $\mathrm{O}(1)-\mathrm{C}(69)-\mathrm{H}(69 \mathrm{C})$ & 109.5 \\
\hline C(58)-C(57)-C(13) & $120.0(9)$ & $\mathrm{H}(69 \mathrm{~A})-\mathrm{C}(69)-\mathrm{H}(69 \mathrm{C})$ & 109.5 \\
\hline C(57)-C(58)-C(59) & $120.5(10)$ & $\mathrm{H}(69 \mathrm{~B})-\mathrm{C}(69)-\mathrm{H}(69 \mathrm{C})$ & 109.5 \\
\hline $\mathrm{C}(57)-\mathrm{C}(58)-\mathrm{H}(58)$ & 119.8 & $\mathrm{O}(1)-\mathrm{C}(70)-\mathrm{H}(70 \mathrm{~A})$ & 109.5 \\
\hline $\mathrm{C}(59)-\mathrm{C}(58)-\mathrm{H}(58)$ & 119.8 & $\mathrm{O}(1)-\mathrm{C}(70)-\mathrm{H}(70 \mathrm{~B})$ & 109.5 \\
\hline C(60)-C(59)-C(58) & $120.0(11)$ & $\mathrm{H}(70 \mathrm{~A})-\mathrm{C}(70)-\mathrm{H}(70 \mathrm{~B})$ & 109.5 \\
\hline $\mathrm{C}(60)-\mathrm{C}(59)-\mathrm{H}(59)$ & 120.0 & $\mathrm{O}(1)-\mathrm{C}(70)-\mathrm{H}(70 \mathrm{C})$ & 109.5 \\
\hline C(58)-C(59)-H(59) & 120.0 & $\mathrm{H}(70 \mathrm{~A})-\mathrm{C}(70)-\mathrm{H}(70 \mathrm{C})$ & 109.5 \\
\hline C(61)-C(60)-C(59) & $118.9(11)$ & $\mathrm{H}(70 \mathrm{~B})-\mathrm{C}(70)-\mathrm{H}(70 \mathrm{C})$ & 109.5 \\
\hline $\mathrm{C}(61)-\mathrm{C}(60)-\mathrm{H}(60)$ & 120.5 & $\mathrm{C}(1)-\mathrm{N}(1)-\mathrm{C}(4)$ & $102.3(7)$ \\
\hline $\mathrm{C}(59)-\mathrm{C}(60)-\mathrm{H}(60)$ & 120.5 & $\mathrm{C}(1)-\mathrm{N}(1)-\mathrm{Zn}$ & $128.6(6)$ \\
\hline $\mathrm{C}(60)-\mathrm{C}(61)-\mathrm{C}(62)$ & $121.8(10)$ & $\mathrm{C}(4)-\mathrm{N}(1)-\mathrm{Zn}$ & $125.2(5)$ \\
\hline $\mathrm{C}(60)-\mathrm{C}(61)-\mathrm{H}(61)$ & 119.1 & $\mathrm{C}(19)-\mathrm{N}(4)-\mathrm{C}(16)$ & $113.4(7)$ \\
\hline $\mathrm{C}(62)-\mathrm{C}(61)-\mathrm{H}(61)$ & 119.1 & $\mathrm{C}(19)-\mathrm{N}(4)-\mathrm{Zn}$ & $121.3(6)$ \\
\hline $\mathrm{C}(61)-\mathrm{C}(62)-\mathrm{C}(57)$ & 121.1(10) & $\mathrm{C}(16)-\mathrm{N}(4)-\mathrm{Zn}$ & $120.1(6)$ \\
\hline $\mathrm{C}(61)-\mathrm{C}(62)-\mathrm{H}(62)$ & 119.4 & $\mathrm{C}(14)-\mathrm{N}(3)-\mathrm{C}(11)$ & $103.3(7)$ \\
\hline $\mathrm{C}(57)-\mathrm{C}(62)-\mathrm{H}(62)$ & 119.4 & $\mathrm{C}(14)-\mathrm{N}(3)-\mathrm{Zn}$ & $129.5(5)$ \\
\hline C(64)-C(63)-C(68) & $114.2(9)$ & $\mathrm{C}(11)-\mathrm{N}(3)-\mathrm{Zn}$ & $124.3(5)$ \\
\hline C(64)-C(63)-C(15) & 126.3(9) & $\mathrm{C}(9)-\mathrm{N}(2)-\mathrm{C}(6)$ & 113.1(8) \\
\hline $\mathrm{C}(68)-\mathrm{C}(63)-\mathrm{C}(15)$ & 119.4(9) & $\mathrm{C}(9)-\mathrm{N}(2)-\mathrm{Zn}$ & $118.3(5)$ \\
\hline $\mathrm{C}(63)-\mathrm{C}(64)-\mathrm{C}(65)$ & $127.2(11)$ & $C(6)-N(2)-Z n$ & $118.8(5)$ \\
\hline
\end{tabular}


Table S5 (Contd.)

\begin{tabular}{|ll|}
\hline \multicolumn{2}{|c|}{ Angle( $\left.{ }^{\circ}\right)$} \\
\hline $\mathrm{B}(70)-\mathrm{O}(1)-\mathrm{C}(69)$ & $46.0(14)$ \\
$\mathrm{C}(70)-\mathrm{O}(1)-\mathrm{Zn}$ & $124.5(15)$ \\
$\mathrm{C}(69)-\mathrm{O}(1)-\mathrm{Zn}$ & $127.8(11)$ \\
$\mathrm{N}(1)-\mathrm{Zn}-\mathrm{N}(3)$ & $156.8(3)$ \\
$\mathrm{N}(1)-\mathrm{Zn}-\mathrm{O}(1)$ & $101.9(3)$ \\
$\mathrm{N}(3)-\mathrm{Zn}-\mathrm{O}(1)$ & $101.2(3)$ \\
$\mathrm{N}(1)-\mathrm{Zn}-\mathrm{N}(4)$ & $88.4(3)$ \\
$\mathrm{N}(3)-\mathrm{Zn}-\mathrm{N}(4)$ & $87.9(3)$ \\
$\mathrm{O}(1)-\mathrm{Zn}-\mathrm{N}(4)$ & $95.1(3)$ \\
$\mathrm{N}(1)-\mathrm{Zn}-\mathrm{N}(2)$ & $88.9(3)$ \\
$\mathrm{N}(3)-\mathrm{Zn}-\mathrm{N}(2)$ & $89.3(3)$ \\
$\mathrm{O}(1)-\mathrm{Zn}-\mathrm{N}(2)$ & $98.8(3)$ \\
$\mathrm{N}(4)-\mathrm{Zn}-\mathrm{N}(2)$ & $166.1(3)$ \\
$\mathrm{C}(17)-\mathrm{C}(18)-\mathrm{C}(19)$ & $110.5(8)$ \\
$\mathrm{C}(17)-\mathrm{C}(18)-\mathrm{Br}(4 \mathrm{~B})$ & $125.2(8)$ \\
$\mathrm{C}(19)-\mathrm{C}(18)-\mathrm{Br}(4 \mathrm{~B})$ & $124.3(7)$ \\
$\mathrm{C}(17)-\mathrm{C}(18)-\mathrm{Br}(4 \mathrm{~A})$ & $126.4(8)$ \\
$\mathrm{C}(19)-\mathrm{C}(18)-\mathrm{Br}(4 \mathrm{~A})$ & $120.8(7)$ \\
$\mathrm{Br}(4 \mathrm{~B})-\mathrm{C}(18)-\mathrm{Br}(4 \mathrm{~A})$ & $13.7(4)$ \\
$\mathrm{C}(18)-\mathrm{C}(17)-\mathrm{C}(16)$ & $112.0(9)$ \\
$\mathrm{C}(18)-\mathrm{C}(17)-\mathrm{Br}(3 \mathrm{~A})$ & $123.0(8)$ \\
$\mathrm{C}(16)-\mathrm{C}(17)-\mathrm{Br}(3 \mathrm{~A})$ & $124.8(8)$ \\
$\mathrm{C}(18)-\mathrm{C}(17)-\mathrm{Br}(3 \mathrm{~B})$ & $123.6(8)$ \\
$\mathrm{C}(16)-\mathrm{C}(17)-\mathrm{Br}(3 \mathrm{~B})$ & $123.4(8)$ \\
$\mathrm{Br}(3 \mathrm{~A})-\mathrm{C}(17)-\mathrm{Br}(3 \mathrm{~B})$ & $13.6(4)$ \\
& \\
\hline
\end{tabular}




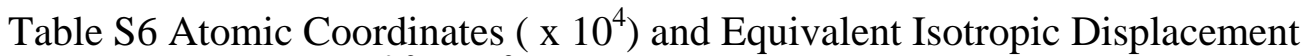
Parameters $\left(\AA^{2} \times 10^{3}\right)$ for $\mathrm{ZnTPP}(\mathrm{Ph})_{4} \mathrm{Br}_{4}\left(\mathrm{CH}_{3} \mathrm{OH}\right) \cdot \mathrm{CH}_{3} \mathrm{OH}$.

$\mathrm{U}(\mathrm{eq})$ is Defined as One Third of the Trace of the Orthogonalized Uij Tensor.

\begin{tabular}{|c|c|c|c|c|}
\hline & $x$ & $\mathrm{y}$ & $\mathrm{z}$ & $\mathrm{U}_{(\mathrm{eq})}$ \\
\hline $\mathrm{C}(1)$ & 9643(7) & $4755(7)$ & 1614(3) & $33(2)$ \\
\hline$C(2)$ & 9135(6) & 5221(7) & $1246(3)$ & $31(2)$ \\
\hline $\mathrm{C}(3)$ & 8148(7) & $5036(7)$ & 1250(3) & $35(3)$ \\
\hline$C(4)$ & $8113(6)$ & $4427(6)$ & 1624(3) & $28(2)$ \\
\hline C(5) & 7246(6) & $3972(6)$ & 1744(3) & $28(2)$ \\
\hline $\mathrm{C}(6)$ & 7159(6) & 3629(6) & 2123(3) & $27(2)$ \\
\hline $\mathrm{C}(7)$ & 6430(6) & $3003(7)$ & 2285(3) & $34(2)$ \\
\hline$C(8)$ & 6551(7) & $3043(7)$ & 2704(3) & $36(3)$ \\
\hline C(9) & 7393(7) & $3683(7)$ & 2861(3) & $33(2)$ \\
\hline$C(10)$ & 7690(6) & $4056(7)$ & $3235(3)$ & $28(2)$ \\
\hline $\mathrm{C}(11)$ & $8627(7)$ & $4510(7)$ & 3358(3) & $32(2)$ \\
\hline $\mathrm{C}(12)$ & 8958(6) & 5103(7) & 3709(3) & $31(2)$ \\
\hline $\mathrm{C}(13)$ & 9933(7) & $5229(7)$ & $3720(3)$ & $30(2)$ \\
\hline$C(14)$ & 10208(6) & $4749(6)$ & $3366(3)$ & $29(2)$ \\
\hline $\mathrm{C}(15)$ & 11194(7) & $4628(7)$ & $3242(3)$ & $39(3)$ \\
\hline $\mathrm{C}(16)$ & 11386(6) & $4408(7)$ & 2857(3) & $39(3)$ \\
\hline $\mathrm{C}(19)$ & 11142(6) & $4476(7)$ & 2100(3) & $36(3)$ \\
\hline$C(20)$ & 10671(6) & 4659(6) & $1700(3)$ & $26(2)$ \\
\hline $\mathrm{C}(21)$ & $11250(6)$ & $4841(7)$ & $1347(3)$ & $28(2)$ \\
\hline $\mathrm{C}(22)$ & 11842(7) & $5621(7)$ & 1337(3) & 41(3) \\
\hline$C(23)$ & 12398(7) & $5832(8)$ & 1020(3) & $50(3)$ \\
\hline$C(24)$ & 12332(8) & $5203(8)$ & $683(4)$ & 63(3) \\
\hline$C(25)$ & 11773(8) & 4383(9) & $677(4)$ & $67(4)$ \\
\hline$C(26)$ & 11252(7) & $4203(7)$ & 1026(3) & $47(3)$ \\
\hline $\mathrm{C}(27)$ & 9502(6) & $5975(7)$ & $963(3)$ & $33(2)$ \\
\hline$C(28)$ & $9725(7)$ & $6852(7)$ & 1132(3) & $46(3)$ \\
\hline$C(29)$ & 9993(8) & $7597(9)$ & $890(4)$ & 73(4) \\
\hline$C(30)$ & 10053(8) & $7445(8)$ & $476(4)$ & 63(3) \\
\hline $\mathrm{C}(31)$ & $9825(8)$ & $6547(8)$ & $289(4)$ & 59(3) \\
\hline$C(32)$ & $9530(8)$ & $5819(8)$ & $541(3)$ & 61(3) \\
\hline C(33) & 7324(7) & $5520(8)$ & $979(3)$ & $46(3)$ \\
\hline$C(34)$ & 7043(8) & $5276(8)$ & $555(4)$ & $60(3)$ \\
\hline $\mathrm{C}(35)$ & $6259(10)$ & 5709(9) & $341(4)$ & $85(4)$ \\
\hline$C(36)$ & $5750(10)$ & 6426(10) & $501(4)$ & $86(4)$ \\
\hline $\mathrm{C}(37)$ & 6021(9) & 6711(9) & $922(4)$ & $77(4)$ \\
\hline C(38) & 6812(8) & $6227(8)$ & 1139(4) & $60(3)$ \\
\hline $\mathrm{C}(39)$ & 6394(7) & $3853(7)$ & 1398(3) & $42(3)$ \\
\hline
\end{tabular}


Table S6 (Contd.).

\begin{tabular}{|ccccc|}
\hline & $\mathrm{x}$ & $\mathrm{y}$ & $\mathrm{z}$ & $\mathrm{U}_{(\mathrm{eq})}$ \\
\hline $\mathrm{C}(40)$ & $6450(9)$ & $3257(8)$ & $1043(4)$ & $65(3)$ \\
$\mathrm{C}(41)$ & $5674(9)$ & $3148(9)$ & $745(4)$ & $72(4)$ \\
$\mathrm{C}(42)$ & $4814(10)$ & $3592(10)$ & $780(4)$ & $85(4)$ \\
$\mathrm{C}(43)$ & $4716(8)$ & $4211(8)$ & $1114(4)$ & $64(3)$ \\
$\mathrm{C}(44)$ & $5504(8)$ & $4321(8)$ & $1426(4)$ & $58(3)$ \\
$\mathrm{C}(45)$ & $7069(7)$ & $3956(8)$ & $3599(3)$ & $43(3)$ \\
$\mathrm{C}(46)$ & $6160(7)$ & $4413(8)$ & $3529(3)$ & $53(3)$ \\
$\mathrm{C}(47)$ & $5572(8)$ & $4320(8)$ & $3855(4)$ & $64(3)$ \\
$\mathrm{C}(48)$ & $5844(9)$ & $3811(8)$ & $4215(4)$ & $68(4)$ \\
$\mathrm{C}(49)$ & $6779(8)$ & $3388(8)$ & $4282(3)$ & $56(3)$ \\
$\mathrm{C}(50)$ & $7351(8)$ & $3492(7)$ & $3964(3)$ & $48(3)$ \\
$\mathrm{C}(51)$ & $8342(7)$ & $5623(7)$ & $3987(3)$ & $35(2)$ \\
$\mathrm{C}(52)$ & $7651(8)$ & $6263(8)$ & $3819(4)$ & $57(3)$ \\
$\mathrm{C}(53)$ & $7088(9)$ & $6801(9)$ & $4058(4)$ & $77(4)$ \\
$\mathrm{C}(54)$ & $7267(9)$ & $6714(9)$ & $4499(4)$ & $69(4)$ \\
$\mathrm{C}(55)$ & $7972(9)$ & $6107(9)$ & $4673(4)$ & $77(4)$ \\
$\mathrm{C}(56)$ & $8480(8)$ & $5560(8)$ & $4420(3)$ & $54(3)$ \\
$\mathrm{C}(57)$ & $10519(7)$ & $5919(7)$ & $4006(3)$ & $38(2)$ \\
$\mathrm{C}(58)$ & $10574(7)$ & $6886(7)$ & $3889(3)$ & $49(3)$ \\
$\mathrm{C}(59)$ & $11090(7)$ & $7552(8)$ & $4165(3)$ & $54(3)$ \\
$\mathrm{C}(60)$ & $11526(8)$ & $7259(8)$ & $4560(4)$ & $61(3)$ \\
$\mathrm{C}(61)$ & $11452(7)$ & $6321(7)$ & $4673(3)$ & $41(3)$ \\
$\mathrm{C}(62)$ & $10967(7)$ & $5655(7)$ & $4403(3)$ & $39(3)$ \\
$\mathrm{C}(63)$ & $11992(6)$ & $4746(6)$ & $3587(3)$ & $26(2)$ \\
$\mathrm{C}(64)$ & $12582(7)$ & $5513(8)$ & $3652(3)$ & $48(3)$ \\
$\mathrm{C}(65)$ & $13296(7)$ & $5664(8)$ & $3989(3)$ & $51(3)$ \\
$\mathrm{C}(66)$ & $13433(8)$ & $4901(8)$ & $4278(3)$ & $53(3)$ \\
$\mathrm{C}(67)$ & $12889(7)$ & $4063(8)$ & $4235(3)$ & $54(3)$ \\
$\mathrm{C}(68)$ & $12157(7)$ & $4027(8)$ & $3903(3)$ & $47(3)$ \\
$\mathrm{C}(69)$ & $9051(18)$ & $1855(18)$ & $2642(14)$ & $72(12)$ \\
$\mathrm{C}(70)$ & $9020(20)$ & $1940(20)$ & $2312(16)$ & $91(14)$ \\
$\mathrm{C}(71)$ & $6461(15)$ & $6165(15)$ & $2478(6)$ & $168(8)$ \\
$\mathrm{N}(1)$ & $9039(5)$ & $4309(5)$ & $1865(2)$ & $29(2)$ \\
$\mathrm{N}(4)$ & $10702(5)$ & $4486(5)$ & $2479(2)$ & $36(2)$ \\
$\mathrm{N}(3)$ & $9432(4)$ & $4355(5)$ & $3113(2)$ & $22(2)$ \\
$\mathrm{N}(2)$ & $7738(5)$ & $3958(5)$ & $2487(2)$ & $30(2)$ \\
$\mathrm{O}(1)$ & $9585(5)$ & $2599(5)$ & $2495(3)$ & $64(2)$ \\
$\mathrm{Zn}$ & $9273(1)$ & $4044(1)$ & $2483(1)$ & $33(1)$ \\
& & & & \\
\hline
\end{tabular}


Table S6 (Contd.).

\begin{tabular}{|lrllr|}
\hline & $\mathrm{x}$ & $\mathrm{y}$ & $\mathrm{z}$ & $\mathrm{U}_{(\mathrm{eq})}$ \\
\hline $\mathrm{O}(2)$ & $7596(13)$ & $6191(12)$ & $2479(5)$ & $237(7)$ \\
$\mathrm{O}(3)$ & $1335(16)$ & $1848(16)$ & $2790(7)$ & $125(5)$ \\
$\mathrm{O}(4)$ & $1121(13)$ & $1960(13)$ & $2188(6)$ & $125(5)$ \\
$\mathrm{Br}(4 \mathrm{~A})$ & $13090(7)$ & $3794(5)$ & $1910(3)$ & $47(2)$ \\
$\mathrm{Br}(4 \mathrm{~B})$ & $13205(6)$ & $4095(5)$ & $1931(3)$ & $44(2)$ \\
$\mathrm{Br}(3 \mathrm{~A})$ & $13551(7)$ & $4097(5)$ & $3006(4)$ & $46(2)$ \\
$\mathrm{Br}(3 \mathrm{~B})$ & $13468(7)$ & $3786(5)$ & $3006(4)$ & $45(2)$ \\
$\mathrm{Br}(2 \mathrm{~A})$ & $5850(7)$ & $2343(6)$ & $3066(4)$ & $47(2)$ \\
$\mathrm{Br}(2 \mathrm{~B})$ & $6007(8)$ & $2100(7)$ & $3049(4)$ & $52(2)$ \\
$\mathrm{Br}(1 \mathrm{~A})$ & $5496(8)$ & $2285(8)$ & $1965(4)$ & $53(2)$ \\
$\mathrm{Br}(1 \mathrm{~B})$ & $5659(8)$ & $2073(7)$ & $1957(4)$ & $49(2)$ \\
$\mathrm{C}(18)$ & $12217(6)$ & $4271(6)$ & $2273(3)$ & $31(2)$ \\
$\mathrm{C}(17)$ & $12329(7)$ & $4234(7)$ & $2678(3)$ & $42(3)$ \\
& & & & \\
\hline
\end{tabular}


Table S7 Bond Lengths $(\AA)$ of NiTPP $(P h)_{4}(\mathrm{CN})_{4}\left(\mathrm{C}_{5} \mathrm{H}_{5} \mathrm{~N}\right)_{2} \cdot 2\left(\mathrm{C}_{5} \mathrm{H}_{5} \mathrm{~N}\right)$.

\begin{tabular}{|c|c|c|c|}
\hline \multicolumn{2}{|c|}{ Bond Distance $(\AA)$} & \multirow{2}{*}{$\begin{array}{l}\text { Bond } \\
\mathrm{C}(22)-\mathrm{C}(23)\end{array}$} & \multirow{2}{*}{$\begin{array}{l}\text { Distance }(\AA) \\
1.340(7)\end{array}$} \\
\hline $\mathrm{C}(1)-\mathrm{N}(1)$ & $1.365(3)$ & & \\
\hline$C(1)-C(10)$ & $1.412(3)$ & $\mathrm{C}(22)-\mathrm{H}(22)$ & 0.9300 \\
\hline$C(1)-C(2)$ & $1.440(3)$ & $\mathrm{C}(23)-\mathrm{C}(24)$ & $1.388(4)$ \\
\hline $\mathrm{C}(2)-\mathrm{C}(3)$ & 1.371(3) & $\mathrm{C}(23)-\mathrm{H}(23)$ & 0.9300 \\
\hline $\mathrm{C}(2)-\mathrm{C}(11)$ & $1.426(3)$ & $\mathrm{C}(24)-\mathrm{H}(24)$ & 0.9300 \\
\hline $\mathrm{C}(3)-\mathrm{C}(12)$ & $1.430(3)$ & $C(25)-C(30)$ & 1.384(3) \\
\hline$C(3)-C(4)$ & $1.435(3)$ & $\mathrm{C}(25)-\mathrm{C}(26)$ & 1.388(3) \\
\hline $\mathrm{C}(4)-\mathrm{N}(1)$ & $1.373(3)$ & $\mathrm{C}(26)-\mathrm{C}(27)$ & $1.391(4)$ \\
\hline$C(4)-C(5)$ & $1.406(3)$ & $\mathrm{C}(26)-\mathrm{H}(26)$ & 0.9300 \\
\hline$C(5)-C(6)$ & $1.402(3)$ & $\mathrm{C}(27)-\mathrm{C}(28)$ & $1.359(5)$ \\
\hline$C(5)-C(13)$ & $1.503(3)$ & $\mathrm{C}(27)-\mathrm{H}(27)$ & 0.9300 \\
\hline$C(6)-N(2)$ & $1.372(3)$ & $\mathrm{C}(28)-\mathrm{C}(29)$ & $1.349(5)$ \\
\hline$C(6)-C(7)$ & $1.463(3)$ & $\mathrm{C}(28)-\mathrm{H}(28)$ & 0.9300 \\
\hline $\mathrm{C}(7)-\mathrm{C}(8)$ & $1.356(3)$ & C(29)-C(30) & $1.383(4)$ \\
\hline $\mathrm{C}(7)-\mathrm{C}(19)$ & 1.493(3) & $\mathrm{C}(29)-\mathrm{H}(29)$ & 0.9300 \\
\hline $\mathrm{C}(8)-\mathrm{C}(9)$ & 1.461(3) & $\mathrm{C}(30)-\mathrm{H}(30)$ & 0.9300 \\
\hline $\mathrm{C}(8)-\mathrm{C}(25)$ & $1.489(3)$ & $\mathrm{C}(31)-\mathrm{C}(36)$ & 1.381(3) \\
\hline $\mathrm{C}(9)-\mathrm{N}(2)$ & $1.377(3)$ & $\mathrm{C}(31)-\mathrm{C}(32)$ & $1.387(3)$ \\
\hline $\mathrm{C}(9)-\mathrm{C}(10) \# 1$ & 1.401(3) & $\mathrm{C}(32)-\mathrm{C}(33)$ & 1.382(3) \\
\hline $\mathrm{C}(10)-\mathrm{C}(9) \# 1$ & $1.401(3)$ & $\mathrm{C}(32)-\mathrm{H}(32)$ & 0.9300 \\
\hline $\mathrm{C}(10)-\mathrm{C}(31)$ & $1.499(3)$ & C(33)-C(34) & $1.368(5)$ \\
\hline $\mathrm{C}(11)-\mathrm{N}(5)$ & $1.145(3)$ & C(33)-H(33) & 0.9300 \\
\hline $\mathrm{C}(12)-\mathrm{N}(6)$ & $1.149(4)$ & $\mathrm{C}(34)-\mathrm{C}(35)$ & $1.377(5)$ \\
\hline $\mathrm{C}(13)-\mathrm{C}(14)$ & 1.378(3) & $\mathrm{C}(34)-\mathrm{H}(34)$ & 0.9300 \\
\hline $\mathrm{C}(13)-\mathrm{C}(18)$ & 1.378(3) & $\mathrm{C}(35)-\mathrm{C}(36)$ & $1.384(4)$ \\
\hline $\mathrm{C}(14)-\mathrm{C}(15)$ & $1.386(4)$ & $\mathrm{C}(35)-\mathrm{H}(35)$ & 0.9300 \\
\hline $\mathrm{C}(14)-\mathrm{H}(14)$ & 0.9300 & $\mathrm{C}(36)-\mathrm{H}(36)$ & 0.9300 \\
\hline $\mathrm{C}(15)-\mathrm{C}(16)$ & $1.361(5)$ & $\mathrm{C}(37)-\mathrm{N}(3)$ & $1.340(3)$ \\
\hline $\mathrm{C}(15)-\mathrm{H}(15)$ & 0.9300 & $\mathrm{C}(37)-\mathrm{C}(38)$ & $1.367(4)$ \\
\hline $\mathrm{C}(16)-\mathrm{C}(17)$ & $1.355(5)$ & $\mathrm{C}(37)-\mathrm{H}(37)$ & 0.9300 \\
\hline $\mathrm{C}(16)-\mathrm{H}(16)$ & 0.9300 & C(38)-C(39) & 1.371(3) \\
\hline $\mathrm{C}(17)-\mathrm{C}(18)$ & $1.388(3)$ & $\mathrm{C}(38)-\mathrm{H}(38)$ & 0.9300 \\
\hline $\mathrm{C}(17)-\mathrm{H}(17)$ & 0.9300 & $\mathrm{C}(39)-\mathrm{C}(38) \#$ & 11.371(3) \\
\hline $\mathrm{C}(18)-\mathrm{H}(18)$ & 0.9300 & $\mathrm{C}(39)-\mathrm{H}(39)$ & $0.99(4)$ \\
\hline $\mathrm{C}(19)-\mathrm{C}(24)$ & $1.377(4)$ & $\mathrm{C}(40)-\mathrm{N}(4)$ & $1.345(3)$ \\
\hline $\mathrm{C}(19)-\mathrm{C}(20)$ & $1.379(4)$ & $\mathrm{C}(40)-\mathrm{C}(41)$ & $1.364(4)$ \\
\hline $\mathrm{C}(20)-\mathrm{C}(21)$ & $1.389(5)$ & $\mathrm{C}(40)-\mathrm{H}(40)$ & 0.9300 \\
\hline $\mathrm{C}(20)-\mathrm{H}(20)$ & 0.9300 & $\mathrm{C}(41)-\mathrm{C}(42)$ & $1.377(4)$ \\
\hline $\mathrm{C}(21)-\mathrm{C}(22)$ & $1.357(7)$ & $\mathrm{C}(41)-\mathrm{H}(41)$ & 0.9300 \\
\hline $\mathrm{C}(21)-\mathrm{H}(21)$ & 0.9300 & $\mathrm{C}(42)-\mathrm{C}(41) \#$ & $11.377(4)$ \\
\hline
\end{tabular}


Table S7 (Contd.).

\begin{tabular}{|ll|}
\hline Bond & Distance $(\AA)$ \\
\hline $\mathrm{C}(42)-\mathrm{H}(42)$ & $1.02(5)$ \\
$\mathrm{C}(43)-\mathrm{C}(44)$ & $1.348(8)$ \\
$\mathrm{C}(43)-\mathrm{N}(7)$ & $1.363(8)$ \\
$\mathrm{C}(43)-\mathrm{H}(43)$ & 0.9300 \\
$\mathrm{C}(44)-\mathrm{C}(45)$ & $1.333(7)$ \\
$\mathrm{C}(44)-\mathrm{H}(44)$ & 0.9300 \\
$\mathrm{C}(45)-\mathrm{C}(46)$ & $1.339(6)$ \\
$\mathrm{C}(45)-\mathrm{H}(45)$ & 0.9300 \\
$\mathrm{C}(46)-\mathrm{C}(47)$ & $1.316(6)$ \\
$\mathrm{C}(46)-\mathrm{H}(46)$ & 0.9300 \\
$\mathrm{C}(47)-\mathrm{N}(7)$ & $1.354(7)$ \\
$\mathrm{C}(47)-\mathrm{H}(47)$ & 0.9300 \\
$\mathrm{~N}(1)-\mathrm{Ni}(1)$ & $2.0505(16)$ \\
$\mathrm{N}(2)-\mathrm{Ni}(1)$ & $2.0885(16)$ \\
$\mathrm{N}(3)-\mathrm{C}(37) \# 1$ & $1.340(3)$ \\
$\mathrm{N}(3)-\mathrm{Ni}(1)$ & $2.189(3)$ \\
$\mathrm{N}(4)-\mathrm{C}(40) \# 1$ & $1.345(3)$ \\
$\mathrm{N}(4)-\mathrm{Ni}(1)$ & $2.242(3)$ \\
$\mathrm{Ni}(1)-\mathrm{N}(1) \# 1$ & $2.0505(16)$ \\
$\mathrm{Ni}(1)-\mathrm{N}(2) \# 1$ & $2.0885(16)$ \\
& \\
\hline
\end{tabular}


Table S8 Bond Angles $\left(^{\circ}\right)$ of NiTPP $(\mathrm{Ph})_{4}(\mathrm{CN})_{4}\left(\mathrm{C}_{5} \mathrm{H}_{5} \mathrm{~N}\right)_{2} \cdot 2\left(\mathrm{C}_{5} \mathrm{H}_{5} \mathrm{~N}\right)$

\begin{tabular}{|c|c|c|c|}
\hline Bond & Angle $\left(^{\circ}\right)$ & Bond & Angle $\left({ }^{\circ}\right)$ \\
\hline$N(1)-C(1)-C(10)$ & 126.83(18) & $\mathrm{C}(17)-\mathrm{C}(16)-\mathrm{C}(15)$ & $119.8(3)$ \\
\hline $\mathrm{N}(1)-\mathrm{C}(1)-\mathrm{C}(2)$ & 108.67(18) & $\mathrm{C}(17)-\mathrm{C}(16)-\mathrm{H}(16)$ & 120.1 \\
\hline $\mathrm{C}(10)-\mathrm{C}(1)-\mathrm{C}(2)$ & 124.50(19) & $\mathrm{C}(15)-\mathrm{C}(16)-\mathrm{H}(16)$ & 120.1 \\
\hline$C(3)-C(2)-C(11)$ & $120.6(2)$ & $\mathrm{C}(16)-\mathrm{C}(17)-\mathrm{C}(18)$ & $120.4(3)$ \\
\hline$C(3)-C(2)-C(1)$ & 107.07(18) & $\mathrm{C}(16)-\mathrm{C}(17)-\mathrm{H}(17)$ & 119.8 \\
\hline$C(11)-C(2)-C(1)$ & $132.2(2)$ & $\mathrm{C}(18)-\mathrm{C}(17)-\mathrm{H}(17)$ & 119.8 \\
\hline $\mathrm{C}(2)-\mathrm{C}(3)-\mathrm{C}(12)$ & $120.5(2)$ & $\mathrm{C}(13)-\mathrm{C}(18)-\mathrm{C}(17)$ & $120.4(3)$ \\
\hline$C(2)-C(3)-C(4)$ & 107.28(18) & C(13)-C(18)-H(18) & 119.8 \\
\hline $\mathrm{C}(12)-\mathrm{C}(3)-\mathrm{C}(4)$ & $131.8(2)$ & $\mathrm{C}(17)-\mathrm{C}(18)-\mathrm{H}(18)$ & 119.8 \\
\hline $\mathrm{N}(1)-\mathrm{C}(4)-\mathrm{C}(5)$ & 127.21(18) & C(24)-C(19)-C(20) & $117.9(2)$ \\
\hline $\mathrm{N}(1)-\mathrm{C}(4)-\mathrm{C}(3)$ & 108.53(18) & $C(24)-C(19)-C(7)$ & $121.3(2)$ \\
\hline$C(5)-C(4)-C(3)$ & 124.25(19) & $C(20)-C(19)-C(7)$ & $120.3(2)$ \\
\hline$C(6)-C(5)-C(4)$ & 125.40(19) & $\mathrm{C}(19)-\mathrm{C}(20)-\mathrm{C}(21)$ & $120.4(4)$ \\
\hline$C(6)-C(5)-C(13)$ & 119.12(18) & $\mathrm{C}(19)-\mathrm{C}(20)-\mathrm{H}(20)$ & 119.8 \\
\hline$C(4)-C(5)-C(13)$ & 115.47(18) & $\mathrm{C}(21)-\mathrm{C}(20)-\mathrm{H}(20)$ & 119.8 \\
\hline $\mathrm{N}(2)-\mathrm{C}(6)-\mathrm{C}(5)$ & 125.01(18) & $\mathrm{C}(22)-\mathrm{C}(21)-\mathrm{C}(20)$ & $120.1(4)$ \\
\hline $\mathrm{N}(2)-\mathrm{C}(6)-\mathrm{C}(7)$ & 109.43(17) & $\mathrm{C}(22)-\mathrm{C}(21)-\mathrm{H}(21)$ & 119.9 \\
\hline$C(5)-C(6)-C(7)$ & 125.56(19) & $\mathrm{C}(20)-\mathrm{C}(21)-\mathrm{H}(21)$ & 119.9 \\
\hline$C(8)-C(7)-C(6)$ & 106.84(18) & $\mathrm{C}(23)-\mathrm{C}(22)-\mathrm{C}(21)$ & $120.6(3)$ \\
\hline $\mathrm{C}(8)-\mathrm{C}(7)-\mathrm{C}(19)$ & 121.09(18) & $\mathrm{C}(23)-\mathrm{C}(22)-\mathrm{H}(22)$ & 119.7 \\
\hline $\mathrm{C}(6)-\mathrm{C}(7)-\mathrm{C}(19)$ & 132.07(19) & $\mathrm{C}(21)-\mathrm{C}(22)-\mathrm{H}(22)$ & 119.7 \\
\hline $\mathrm{C}(7)-\mathrm{C}(8)-\mathrm{C}(9)$ & 107.30(18) & $\mathrm{C}(22)-\mathrm{C}(23)-\mathrm{C}(24)$ & $120.1(4)$ \\
\hline C(7)-C(8)-C(25) & 121.93(19) & $\mathrm{C}(22)-\mathrm{C}(23)-\mathrm{H}(23)$ & 119.9 \\
\hline$C(9)-C(8)-C(25)$ & 130.70(19) & $\mathrm{C}(24)-\mathrm{C}(23)-\mathrm{H}(23)$ & 119.9 \\
\hline N(2)-C(9)-C(10)\#1 & $125.00(18)$ & $\mathrm{C}(19)-\mathrm{C}(24)-\mathrm{C}(23)$ & $120.9(3)$ \\
\hline $\mathrm{N}(2)-\mathrm{C}(9)-\mathrm{C}(8)$ & 109.10(17) & $\mathrm{C}(19)-\mathrm{C}(24)-\mathrm{H}(24)$ & 119.5 \\
\hline C(10)\#1-C(9)-C(8) & 125.90(19) & $\mathrm{C}(23)-\mathrm{C}(24)-\mathrm{H}(24)$ & 119.5 \\
\hline C(9)\#1-C(10)-C(1) & 126.08(19) & $C(30)-C(25)-C(26)$ & $118.2(2)$ \\
\hline $\mathrm{C}(9) \# 1-\mathrm{C}(10)-\mathrm{C}(31)$ & 118.80(18) & $C(30)-C(25)-C(8)$ & $121.5(2)$ \\
\hline $\mathrm{C}(1)-\mathrm{C}(10)-\mathrm{C}(31)$ & 115.06(17) & C(26)-C(25)-C(8) & $120.2(2)$ \\
\hline $\mathrm{N}(5)-\mathrm{C}(11)-\mathrm{C}(2)$ & 172.6(3) & C(25)-C(26)-C(27) & $120.2(3)$ \\
\hline N(6)-C(12)-C(3) & 172.3(3) & $\mathrm{C}(25)-\mathrm{C}(26)-\mathrm{H}(26)$ & 119.9 \\
\hline C(14)-C(13)-C(18) & $118.5(2)$ & $\mathrm{C}(27)-\mathrm{C}(26)-\mathrm{H}(26)$ & 119.9 \\
\hline$C(14)-C(13)-C(5)$ & $121.2(2)$ & $\mathrm{C}(28)-\mathrm{C}(27)-\mathrm{C}(26)$ & $120.2(3)$ \\
\hline $\mathrm{C}(18)-\mathrm{C}(13)-\mathrm{C}(5)$ & $120.2(2)$ & $\mathrm{C}(28)-\mathrm{C}(27)-\mathrm{H}(27)$ & 119.9 \\
\hline C(13)-C(14)-C(15) & $120.3(3)$ & $\mathrm{C}(26)-\mathrm{C}(27)-\mathrm{H}(27)$ & 119.9 \\
\hline C(13)-C(14)-H(14) & 119.9 & $\mathrm{C}(29)-\mathrm{C}(28)-\mathrm{C}(27)$ & $120.4(3)$ \\
\hline C(15)-C(14)-H(14) & 119.9 & $\mathrm{C}(29)-\mathrm{C}(28)-\mathrm{H}(28)$ & 119.8 \\
\hline C(16)-C(15)-C(14) & 120.5(3) & $\mathrm{C}(27)-\mathrm{C}(28)-\mathrm{H}(28)$ & 119.8 \\
\hline C(16)-C(15)-H(15) & 119.7 & C(28)-C(29)-C(30) & $120.6(3)$ \\
\hline C(14)-C(15)-H(15) & 119.7 & C(28)-C(29)-H(29) & 119.7 \\
\hline
\end{tabular}


Table S8 (Contd.).

\begin{tabular}{|c|c|c|c|}
\hline Bond & Angle $\left(^{\circ}\right)$ & Bond & Angle $\left(^{\circ}\right)$ \\
\hline C(30)-C(29)-H(29) & 119.7 & $\mathrm{~N}(7)-\mathrm{C}(43)-\mathrm{H}(43)$ & 118.3 \\
\hline C(29)-C(30)-C(25) & $120.5(3)$ & C(45)-C(44)-C(43) & 118.1(5) \\
\hline $\mathrm{C}(29)-\mathrm{C}(30)-\mathrm{H}(30)$ & 119.8 & $\mathrm{C}(45)-\mathrm{C}(44)-\mathrm{H}(44)$ & 121.0 \\
\hline $\mathrm{C}(25)-\mathrm{C}(30)-\mathrm{H}(30)$ & 119.8 & $\mathrm{C}(43)-\mathrm{C}(44)-\mathrm{H}(44)$ & 121.0 \\
\hline $\mathrm{C}(36)-\mathrm{C}(31)-\mathrm{C}(32)$ & $119.4(2)$ & $C(44)-C(45)-C(46)$ & $120.4(5)$ \\
\hline $\mathrm{C}(36)-\mathrm{C}(31)-\mathrm{C}(10)$ & $122.1(2)$ & $\mathrm{C}(44)-\mathrm{C}(45)-\mathrm{H}(45)$ & 119.8 \\
\hline $\mathrm{C}(32)-\mathrm{C}(31)-\mathrm{C}(10)$ & $118.5(2)$ & $\mathrm{C}(46)-\mathrm{C}(45)-\mathrm{H}(45)$ & 119.8 \\
\hline $\mathrm{C}(33)-\mathrm{C}(32)-\mathrm{C}(31)$ & 120.3(3) & $\mathrm{C}(47)-\mathrm{C}(46)-\mathrm{C}(45)$ & $120.3(5)$ \\
\hline C(33)-C(32)-H(32) & 119.8 & $\mathrm{C}(47)-\mathrm{C}(46)-\mathrm{H}(46)$ & 119.8 \\
\hline C(31)-C(32)-H(32) & 119.8 & $\mathrm{C}(45)-\mathrm{C}(46)-\mathrm{H}(46)$ & 119.8 \\
\hline $\mathrm{C}(34)-\mathrm{C}(33)-\mathrm{C}(32)$ & 119.8(3) & $\mathrm{C}(46)-\mathrm{C}(47)-\mathrm{N}(7)$ & $122.8(5)$ \\
\hline C(34)-C(33)-H(33) & 120.1 & $\mathrm{C}(46)-\mathrm{C}(47)-\mathrm{H}(47)$ & 118.6 \\
\hline C(32)-C(33)-H(33) & 120.1 & $\mathrm{~N}(7)-\mathrm{C}(47)-\mathrm{H}(47)$ & 118.6 \\
\hline C(33)-C(34)-C(35) & $120.5(2)$ & $\mathrm{C}(1)-\mathrm{N}(1)-\mathrm{C}(4)$ & 108.41(16) \\
\hline C(33)-C(34)-H(34) & 119.7 & C(1)-N(1)-Ni(1) & $125.65(14)$ \\
\hline C(35)-C(34)-H(34) & 119.7 & $\mathrm{C}(4)-\mathrm{N}(1)-\mathrm{Ni}(1)$ & $125.77(13)$ \\
\hline $\mathrm{C}(34)-\mathrm{C}(35)-\mathrm{C}(36)$ & 119.9(3) & $\mathrm{C}(6)-\mathrm{N}(2)-\mathrm{C}(9)$ & 107.32(16) \\
\hline $\mathrm{C}(34)-\mathrm{C}(35)-\mathrm{H}(35)$ & 120.0 & $\mathrm{C}(6)-\mathrm{N}(2)-\mathrm{Ni}(1)$ & 126.74(13) \\
\hline $\mathrm{C}(36)-\mathrm{C}(35)-\mathrm{H}(35)$ & 120.0 & $\mathrm{C}(9)-\mathrm{N}(2)-\mathrm{Ni}(1)$ & $125.90(13)$ \\
\hline C(31)-C(36)-C(35) & $120.0(3)$ & C(37)\#1-N(3)-C(37) & $116.5(3)$ \\
\hline $\mathrm{C}(31)-\mathrm{C}(36)-\mathrm{H}(36)$ & 120.0 & C(37)\#1-N(3)-Ni(1) & $121.73(14)$ \\
\hline $\mathrm{C}(35)-\mathrm{C}(36)-\mathrm{H}(36)$ & 120.0 & C(37)-N(3)-Ni(1) & $121.73(14)$ \\
\hline N(3)-C(37)-C(38) & $123.3(2)$ & C(40)\#1-N(4)-C(40) & $115.4(3)$ \\
\hline N(3)-C(37)-H(37) & 118.4 & $\mathrm{C}(40) \# 1-\mathrm{N}(4)-\mathrm{Ni}(1)$ & $122.28(15)$ \\
\hline C(38)-C(37)-H(37) & 118.4 & $\mathrm{C}(40)-\mathrm{N}(4)-\mathrm{Ni}(1)$ & $122.28(15)$ \\
\hline C(37)-C(38)-C(39) & 119.3(3) & $\mathrm{C}(47)-\mathrm{N}(7)-\mathrm{C}(43)$ & $114.9(5)$ \\
\hline C(37)-C(38)-H(38) & 120.3 & $\mathrm{~N}(1)-\mathrm{Ni}(1)-\mathrm{N}(1) \# 1$ & $179.26(10)$ \\
\hline C(39)-C(38)-H(38) & 120.3 & N(1)-Ni(1)-N(2) & 89.67(7) \\
\hline C(38)\#1-C(39)-C(38) & $118.2(4)$ & $\mathrm{N}(1) \# 1-\mathrm{Ni}(1)-\mathrm{N}(2)$ & $90.33(7)$ \\
\hline C(38)\#1-C(39)-H(39) & 120.88(19) & $\mathrm{N}(1)-\mathrm{Ni}(1)-\mathrm{N}(2) \# 1$ & $90.33(7)$ \\
\hline C(38)-C(39)-H(39) & 120.88(19) & $\mathrm{N}(1) \# 1-\mathrm{Ni}(1)-\mathrm{N}(2) \# 1$ & $89.67(7)$ \\
\hline N(4)-C(40)-C(41) & $123.9(2)$ & $\mathrm{N}(2)-\mathrm{Ni}(1)-\mathrm{N}(2) \# 1$ & 179.67(10) \\
\hline N(4)-C(40)-H(40) & 118.0 & N(1)-Ni(1)-N(3) & 89.63(5) \\
\hline $\mathrm{C}(41)-\mathrm{C}(40)-\mathrm{H}(40)$ & 118.0 & N(1)\#1-Ni(1)-N(3) & 89.63(5) \\
\hline $\mathrm{C}(40)-\mathrm{C}(41)-\mathrm{C}(42)$ & 119.6(3) & $\mathrm{N}(2)-\mathrm{Ni}(1)-\mathrm{N}(3)$ & 89.84(5) \\
\hline $\mathrm{C}(40)-\mathrm{C}(41)-\mathrm{H}(41)$ & 120.2 & $\mathrm{~N}(2) \# 1-\mathrm{Ni}(1)-\mathrm{N}(3)$ & $89.84(5)$ \\
\hline $\mathrm{C}(42)-\mathrm{C}(41)-\mathrm{H}(41)$ & 120.2 & $\mathrm{~N}(1)-\mathrm{Ni}(1)-\mathrm{N}(4)$ & $90.37(5)$ \\
\hline C(41)\#1-C(42)-C(41) & $117.4(4)$ & $\mathrm{N}(1) \# 1-\mathrm{Ni}(1)-\mathrm{N}(4)$ & $90.37(5)$ \\
\hline C(41)\#1-C(42)-H(42) & $121.3(2)$ & $\mathrm{N}(2)-\mathrm{Ni}(1)-\mathrm{N}(4)$ & $90.16(5)$ \\
\hline $\mathrm{C}(41)-\mathrm{C}(42)-\mathrm{H}(42)$ & $121.3(2)$ & N(2)\#1-Ni(1)-N(4) & $90.16(5)$ \\
\hline $\mathrm{C}(44)-\mathrm{C}(43)-\mathrm{N}(7)$ & $123.4(6)$ & N(3)-Ni(1)-N(4) & $180.000(1)$ \\
\hline $\mathrm{C}(44)-\mathrm{C}(43)-\mathrm{H}(43)$ & 118.3 & & \\
\hline
\end{tabular}


Table S9 Atomic Coordinates (x $\left.10^{4}\right)$ and Equivalent Isotropic Displacement Parameters $\left(\AA^{2} \times 10^{3}\right)$ for NiTPP $(\mathrm{Ph})_{4}(\mathrm{CN})_{4}\left(\mathrm{C}_{5} \mathrm{H}_{5} \mathrm{~N}\right)_{2} \cdot 2\left(\mathrm{C}_{5} \mathrm{H}_{5} \mathrm{~N}\right)$. U(eq) is Defined as one Third of the Trace of the Orthogonalized Uij Tensor.

\begin{tabular}{|lllll|}
\hline & $\mathrm{x}$ & $\mathrm{y}$ & $\mathrm{z}$ & $\mathrm{U}_{(\mathrm{eq})}$ \\
\hline $\mathrm{C}(1)$ & $4722(1)$ & $1251(1)$ & $6017(1)$ & $39(1)$ \\
$\mathrm{C}(2)$ & $4114(1)$ & $1289(1)$ & $5351(1)$ & $44(1)$ \\
$\mathrm{C}(3)$ & $3435(1)$ & $1291(1)$ & $5424(1)$ & $47(1)$ \\
$\mathrm{C}(4)$ & $3621(1)$ & $1229(1)$ & $6130(1)$ & $40(1)$ \\
$\mathrm{C}(5)$ & $3074(1)$ & $1200(1)$ & $6406(1)$ & $39(1)$ \\
$\mathrm{C}(6)$ & $3239(1)$ & $1190(1)$ & $7095(1)$ & $38(1)$ \\
$\mathrm{C}(7)$ & $2672(1)$ & $1154(1)$ & $7384(1)$ & $39(1)$ \\
$\mathrm{C}(8)$ & $3067(1)$ & $1176(1)$ & $8062(1)$ & $39(1)$ \\
$\mathrm{C}(9)$ & $3881(1)$ & $1219(1)$ & $8203(1)$ & $38(1)$ \\
$\mathrm{C}(10)$ & $5512(1)$ & $1253(1)$ & $6157(1)$ & $38(1)$ \\
$\mathrm{C}(11)$ & $4116(2)$ & $1291(2)$ & $4698(1)$ & $59(1)$ \\
$\mathrm{C}(12)$ & $2724(2)$ & $1421(2)$ & $4858(1)$ & $74(1)$ \\
$\mathrm{C}(13)$ & $2250(1)$ & $1183(1)$ & $5903(1)$ & $42(1)$ \\
$\mathrm{C}(14)$ & $1795(2)$ & $1790(2)$ & $5768(2)$ & $71(1)$ \\
$\mathrm{C}(15)$ & $1035(2)$ & $1760(2)$ & $5307(2)$ & $95(1)$ \\
$\mathrm{C}(16)$ & $730(2)$ & $1134(2)$ & $4981(2)$ & $79(1)$ \\
$\mathrm{C}(17)$ & $1167(2)$ & $529(2)$ & $5117(1)$ & $72(1)$ \\
$\mathrm{C}(18)$ & $1930(1)$ & $550(1)$ & $5575(1)$ & $57(1)$ \\
$\mathrm{C}(19)$ & $1817(1)$ & $1105(1)$ & $7068(1)$ & $47(1)$ \\
$\mathrm{C}(20)$ & $1377(2)$ & $1691(2)$ & $7077(2)$ & $91(1)$ \\
$\mathrm{C}(21)$ & $588(2)$ & $1621(3)$ & $6862(2)$ & $128(2)$ \\
$\mathrm{C}(22)$ & $243(2)$ & $976(4)$ & $6633(2)$ & $122(2)$ \\
$\mathrm{C}(23)$ & $659(2)$ & $398(3)$ & $6623(2)$ & $100(1)$ \\
$\mathrm{C}(24)$ & $1448(2)$ & $456(2)$ & $6843(1)$ & $66(1)$ \\
$\mathrm{C}(25)$ & $2675(1)$ & $1123(1)$ & $8526(1)$ & $44(1)$ \\
$\mathrm{C}(26)$ & $2413(2)$ & $461(2)$ & $8642(1)$ & $60(1)$ \\
$\mathrm{C}(27)$ & $2004(2)$ & $418(2)$ & $9041(2)$ & $76(1)$ \\
$\mathrm{C}(28)$ & $1864(2)$ & $1024(2)$ & $9323(2)$ & $82(1)$ \\
$\mathrm{C}(29)$ & $2110(2)$ & $1673(2)$ & $9211(2)$ & $79(1)$ \\
$\mathrm{C}(30)$ & $2512(1)$ & $1731(2)$ & $8811(1)$ & $59(1)$ \\
$\mathrm{C}(31)$ & $5693(1)$ & $1333(1)$ & $5554(1)$ & $42(1)$ \\
$\mathrm{C}(32)$ & $5691(1)$ & $2020(1)$ & $5300(1)$ & $53(1)$ \\
$\mathrm{C}(33)$ & $5853(2)$ & $2117(2)$ & $4744(1)$ & $71(1)$ \\
$\mathrm{C}(34)$ & $6023(2)$ & $1533(2)$ & $4446(1)$ & $81(1)$ \\
$\mathrm{C}(35)$ & $6020(2)$ & $848(2)$ & $4688(1)$ & $75(1)$ \\
$\mathrm{C}(36)$ & $5847(1)$ & $746(2)$ & $5239(1)$ & $57(1)$ \\
$\mathrm{C}(37)$ & $4784(1)$ & $-339(1)$ & $7916(1)$ & $50(1)$ \\
$\mathrm{C}(38)$ & $4775(2)$ & $-1076(2)$ & $7927(1)$ & $61(1)$ \\
$\mathrm{C}(39)$ & 5000 & $-1456(2)$ & 7500 & $66(1)$ \\
\hline & & & & \\
\end{tabular}


Table S9 (Contd.).

\begin{tabular}{|lllll|}
\hline & $\mathrm{x}$ & $\mathrm{y}$ & $\mathrm{z}$ & $\mathrm{U}_{(\mathrm{eq})}$ \\
\hline $\mathrm{C}(40)$ & $5480(1)$ & $2820(1)$ & $8027(1)$ & $58(1)$ \\
$\mathrm{C}(41)$ & $5495(2)$ & $3556(2)$ & $8046(1)$ & $70(1)$ \\
$\mathrm{C}(42)$ & 5000 & $3942(2)$ & 7500 & $73(1)$ \\
$\mathrm{C}(43)$ & $6495(4)$ & $3329(3)$ & $6881(4)$ & $140(2)$ \\
$\mathrm{C}(44)$ & $7109(4)$ & $3444(3)$ & $7465(3)$ & $131(2)$ \\
$\mathrm{C}(45)$ & $7743(3)$ & $3709(3)$ & $7437(2)$ & $119(2)$ \\
$\mathrm{C}(46)$ & $7765(3)$ & $3844(2)$ & $6842(2)$ & $107(1)$ \\
$\mathrm{C}(47)$ & $7155(4)$ & $3732(2)$ & $6279(2)$ & $112(2)$ \\
$\mathrm{N}(1)$ & $4405(1)$ & $1216(1)$ & $6473(1)$ & $39(1)$ \\
$\mathrm{N}(2)$ & $3962(1)$ & $1220(1)$ & $7604(1)$ & $39(1)$ \\
$\mathrm{N}(3)$ & 5000 & $41(1)$ & 7500 & $45(1)$ \\
$\mathrm{N}(4)$ & 5000 & $2433(1)$ & 7500 & $45(1)$ \\
$\mathrm{N}(6)$ & $2202(2)$ & $1538(2)$ & $4363(2)$ & $117(1)$ \\
$\mathrm{N}(5)$ & $4033(2)$ & $1289(2)$ & $4147(1)$ & $85(1)$ \\
$\mathrm{N}(7)$ & $6481(3)$ & $3490(3)$ & $6266(3)$ & $145(2)$ \\
$\mathrm{Ni}(1)$ & 5000 & $1223(1)$ & 7500 & $37(1)$ \\
\hline
\end{tabular}

